\title{
Pitx controls amphioxus asymmetric morphogenesis by promoting left-side development and repressing right-side formation
}

\author{
Chaofan Xing ${ }^{1 \dagger}$, Rongrong Pan ${ }^{1 \dagger}$, Guangwei Hu ${ }^{1,2}$, Xian Liu', Yiquan Wang ${ }^{1}$ and Guang $\mathrm{Li}^{i^{*}}$ (D)
}

\begin{abstract}
Background: Left-right (LR) asymmetry is an essential feature of bilateral animals. Studies in vertebrates show that $L R$ asymmetry formation comprises three major steps: symmetry breaking, asymmetric gene expression, and LR morphogenesis. Although much progress has been made in the first two events, mechanisms underlying asymmetric morphogenesis remain largely unknown due to the complex developmental processes deployed by vertebrate organs.

Results: We here addressed this question by studying Pitx gene function in the basal chordate amphioxus whose asymmetric organogenesis, unlike that in vertebrates, occurs essentially in situ and does not rely on cell migration. Pitx null mutation in amphioxus causes loss of all left-sided organs and incomplete ectopic formation of all right-sided organs on the left side, whereas Pitx partial loss-of-function leads to milder phenotypes with only some LR organs lost or ectopically formed. At the N1 to N3 stages, Pitx expression is gradually expanded from the dorsal anterior domain to surrounding regions. This leads to activation of genes like $L h \times 3$ and/or Prop 1 and Pit, which are essential for left-side organs, and downregulation of genes like Hex and/or Nkx2.1 and FoxE4, which are required for right-side organs to form ectopically on the left side. In Pitx mutants, the left-side expressed genes are not activated, while the right-side genes fail to decrease expression on the left side. In contrast, in embryos overexpressing Pitx genes, the left-side genes are induced ectopically on the right side, and the right-side genes are inhibited. Several Pitx binding sites are identified in the upstream sequences of the left-side and right-side genes which are essential for activation of the former and repression of the latter by Pitx.
\end{abstract}

\footnotetext{
* Correspondence: Guangli@xmu.edu.cn

${ }^{+}$Chaofan Xing and Rongrong Pan contributed equally to this work. ${ }^{1}$ State Key Laboratory of Cellular Stress Biology, School of Life Sciences, Xiamen University, Xiangan District, Xiamen 361102, Fujian, China Full list of author information is available at the end of the article
}

(c) The Author(s). 2021 Open Access This article is licensed under a Creative Commons Attribution 4.0 International License, which permits use, sharing, adaptation, distribution and reproduction in any medium or format, as long as you give appropriate credit to the original author(s) and the source, provide a link to the Creative Commons licence, and indicate if changes were made. The images or other third party material in this article are included in the article's Creative Commons licence, unless indicated otherwise in a credit line to the material. If material is not included in the article's Creative Commons licence and your intended use is not permitted by statutory regulation or exceeds the permitted use, you will need to obtain permission directly from the copyright holder. To view a copy of this licence, visit http://creativecommons.org/licenses/by/4.0/ The Creative Commons Public Domain Dedication waiver (http://creativecommons.org/publicdomain/zero/1.0/) applies to the data made available in this article, unless otherwise stated in a credit line to the data. 
Conclusions: Our results demonstrate that (1) Pitx is a major (although not the only) determinant of asymmetric morphogenesis in amphioxus, (2) the development of different LR organs have distinct requirements for Pitx activity, and (3) Pitx controls amphioxus LR morphogenesis probably through inducing left-side organs and inhibiting right-side organs directly. These findings show much more dependence of LR organogenesis on Pitx in amphioxus than in vertebrates. They also provide insight into the molecular developmental mechanism of some vertebrate LR organs like the lungs and atria, since they show a right-isomerism phenotype in Pitx2 knockout mice like right-sided organs in Pitx mutant amphioxus. Our results also explain why some organs like the adenohypophysis are asymmetrically located in amphioxus but symmetrically positioned in vertebrates.

\section{Background}

While vertebrates exhibit external left-right (LR) symmetry, their visceral organs are LR asymmetric in terms of shape, size, position, and rotation direction [1]. For example, the human heart, spleen, and stomach are offset to the left while the gall bladder and liver sit to the right, and the left and right lung and kidney are of different size and shape [2]. Establishment of LR asymmetry is highly conserved among vertebrates and comprises three major steps: symmetry breaking, asymmetric gene expression, and LR morphogenesis $[1,3]$. Symmetry breaking in most vertebrate groups is initiated by a cilia-driven fluid flow present in the LR organizer of early somite stage embryos [4]. Influenced by this flow, the Dand5 gene first exhibits an asymmetric expression pattern $(\mathrm{R}>\mathrm{L})$ through unilateral mRNA decay in cells around the LR organizer. The Dand5 protein is a Nodal signaling inhibitor, thus its right side-biased expression leads to $\mathrm{R}<\mathrm{L}$ asymmetric Nodal signaling at the LR organizer. The Nodal signaling pathway is transferred further to the left lateral plate mesoderm (LPM) from the organizer and induces asymmetric expression of Nodal itself, followed by Lefty and Pitx2 [5].

Although progress has been made in elucidating the upstream patterning events, mechanisms controlling LR morphogenesis of visceral organs remain largely unknown [3]. This has been complicated by the complex developmental process of vertebrate LR organs, which involves many asymmetric cell behaviors and coordination of various organs within the cavity [3]. Among genes induced by the Nodal signaling pathway, Pitx2 encodes a homeodomain-containing transcription factor and its asymmetric expression persists in developing visceral organs after asymmetric Nodal expression disappears [6]. Therefore, Pitx2 was thought to play an essential role in asymmetric organogenesis. Consistently, knockout of Pitx2 in mice or knockdown in chicken and frogs cause laterality defects in many visceral organs, including randomized positioning of several visceral organs and right isomerism of the lungs and atrium [7-10]. In mice, development of different $L R$ organs requires distinct dosages of Pitx2 activity [11-13]. During mouse and chicken gut development, Pitx2 induces asymmetric cell condensation of dorsal mesentery (DM) attached to the gut by targeting the non-canonical Wnt signaling mediator Daam2, resulting in the tilting of the gut tube toward the left [14]. However, Pitx2 appears to play a very limited role in zebrafish LR development, since its mutation or knockdown causes either no or very weak LR defects [15-17]. This indicates different requirements of Pitx2 for LR development among vertebrates. While the development of most LR asymmetric organs relies on both Nodal and Pitx2, some asymmetric morphogenetic events depend on Nodal but not Pitx2. For example, in mice, Nodal deficiency randomizes directions of heart looping, axial rotation, and stomach sidedness [18], but none of these events are affected in Pitx2 null mutants [11, 12]. This indicates genes other than Pitx2 downstream of the Nodal signaling pathway also play certain roles in LR morphogenesis in vertebrates [19]. Moreover, a recent study suggests that heart looping in zebrafish, chick, and mouse is controlled by a BMP-Prrx1/Snail1 cascade on the right side [20, 21]. However, this suggestion has been questioned by a later study, which shows that neither single nor double mutants of Prrx $1 a$ and Prrx $1 b$ genes cause LR defects in zebrafish embryos [22]. This leaves it uncertain as to whether vertebrates have a right-side determinant signal.

Orthologues of the Nodal and Pitx2 gene have been identified in several diverse groups of invertebrate bilaterians [23]. In most of these animals, the two genes show asymmetric expression along the LR axis. In addition, inhibiting the Nodal signaling pathway also abolishes Pitx expression and leads to defects in LR establishment [24-28]. This demonstrates a certain level of conservation for the genetic control of LR development between invertebrates and vertebrates. However, as in vertebrates, molecular mechanisms underlying LR morphogenesis in invertebrates are largely unknown. In addition, whether Pitx is required for LR asymmetry in invertebrates has not been functionally tested. To address these questions, we here analyzed Pitx gene function in the LR morphogenesis of the cephalochordate amphioxus. Amphioxus shows pronounced LR asymmetries in its pharynx at larval stages, with the mouth, preoral pit and the duct of the club-shaped gland (hereafter refer to as CSG) forming on the left side, and the gill slits, endostyle, and the glandular region of the CSG on the right side $[28,29]$. The arrangement of the somites and peripheral nerves is also asymmetric in 
amphioxus beginning from the N3 stage. Importantly, unlike vertebrates, LR organogenesis of amphioxus occurs essentially in situ and does not rely on asymmetric cell behaviors, and knockout of genes essential for early LR patterning in amphioxus leads to simply either two-right-side (right isomerism) or two-left-side (left isomerism) phenotypes [24, 28, 30-33]. Despite these differences, the molecular mechanisms underlying the early development of LR asymmetry in amphioxus are very similar to that in vertebrates, as they also require cilia movement and Dand5, Nodal, and Lefty genes [24, 28, 30-32]. This, together with its position among chordates, indicates that amphioxus is a promising organism for understanding mechanisms and evolution of vertebrate LR morphogenesis.

We here show that Pitx is a major (although not the only) determinant in amphioxus LR morphogenesis, with its mutation causing loss of all left-side organs and ectopic formation of all right-side organs on the left side. We further demonstrate that different LR organs of amphioxus embryos have distinct requirements for Pitx dosage. Finally, we show that Pitx executes its functions in amphioxus LR morphogenesis probably through directly inducing left-side organs and inhibiting right-side structures. These results demonstrate a conserved role for Pitx in LR development among chordates, and also shed important insights into the molecular mechanisms underlying animal LR morphogenesis.

\section{Results}

Pitx encodes two isoforms with different expression patterns in amphioxus embryos

Vertebrates have three highly conserved Pitx2 mRNA isoforms: Pitx2a, Pitx2b, and Pitx2c. Among them, Pitx $2 c$ is asymmetrically expressed, while the other two, transcribed from an alternative promoter, are not [34]. Two corresponding isoforms (Pitxa/b and Pitxc) have also been reported in tunicate embryos [35]. Our analysis revealed two isoforms for the amphioxus Pitx gene that were transcribed from different transcription initiation sites and were interrupted by two and three introns respectively (Fig. 1a). As they share similar exon/ intron structures to that of the two tunicate Pitx isoforms, we named them Pitxa/b and Pitxc respectively.

The expression patterns of Pitxa/b and Pitxc in amphioxus embryos was analyzed using in situ hybridization with probes corresponding to their specific exons (Fig. $\left.1 \mathrm{~b}-\mathrm{g}^{\prime}\right)$. Pitxa/b expression was first detected in two patches of cells in the neural ectoderm of embryos at $\mathrm{N} 4$ stage (Fig. 1c, c'). Double in situ analysis revealed that these Pitxa/b-positive cells were located more posteriorly than the dorsal Hesse organ expressing Mop (Additional file 1: Fig. S1). At T1 stage, a new Pitxa/b expression domain was observed in the posterior half of the preoral pit (Fig. 1d, d'). Pitxc expression was detected in the left dorsal side of embryos at N1 stage (Fig. 1e, e'), and at N4 and T1 stages, Pitxc was strongly expressed in the anterior left pharyngeal region and weakly in the left side of the tailbud (Fig. 1f-g'). These results

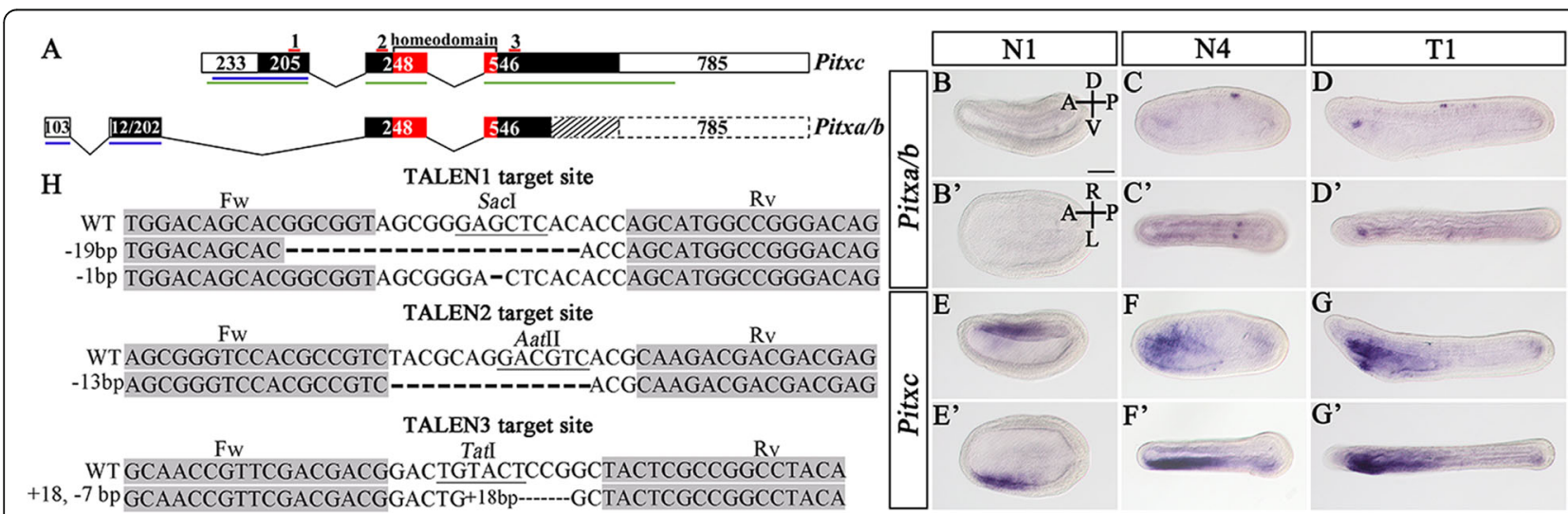

Fig. 1 Expression patterns of amphioxus Pitx isoforms and TALEN target sites of Pitx gene. a Amphioxus Pitx isoforms. White boxes represent untranslated regions (UTR) and black and red boxes (Pitx homeodomain) represent coding sequences. Broken lines indicate introns. We obtained the partial sequence of Pitxa/b according to available amphioxus EST data and the predicted remaining sequence is supplemented as a dotted slashed box (coding sequence) and dotted white box (UTR). $\mathbf{b}-\mathbf{b}^{\prime}$ Expression of Pitxa/b in left lateral (b-d) and dorsal ( $\left.\mathbf{b}^{\prime}-\mathbf{d}^{\prime}\right)$ views. $\mathbf{e}-\mathbf{g}^{\prime}$ Expression of Pitxc in left lateral $(\mathbf{e}-\mathbf{g})$ and dorsal $\left(\mathbf{e}^{\prime} \mathbf{-}-\mathbf{g}^{\prime}\right)$ views. Blue lines in a show the location of Pitxc or Pitxa/b probes and green lines in a show the location of Pitx probe used for whole-mount in situ hybridization (WISH). $\mathbf{h}$ Numbers 1, 2, and 3 in a show the location of TALEN target sites in Pitxc and Pitxa/b. Binding sites for TALEN pairs [forward (Fw) and reverse (Rv)] used in this study are highlighted in gray. The Sacl, Aatll, and Tatl restriction site in the spacer is underlined respectively. WT, wild type sequence. $A$, anterior; P, posterior; D, dorsal; V, ventral; L, left side; R; right side. Scale bars in $\left(\mathbf{b}-\mathbf{g}^{\prime}\right)$ are $50 \mu \mathrm{m}$ 
demonstrated that Pitx in amphioxus also encodes different isoforms with distinct expression patterns, similar to Pitx in vertebrates and tunicates.

\section{Pitx mutation affects amphioxus development while Pitxc mutation does not}

To study the function of Pitx in amphioxus development, we generated Pitx mutants using the TALENmediated genome editing method [36, 37]. Three pairs of TALEN constructs (TALEN1-3) were assembled, which targeted the first coding exon of Pitxc isoform and two other coding exons shared by Pitxc and Pitxa/b (Fig. 1h). Injection of TALEN mRNAs into amphioxus embryos revealed efficient mutation (30-50\%) at each of the target sites (Additional file 1: Fig. S2). Using these TALENs, we obtained four Pitx mutant lines (19-bp and 1-bp deletion for TALEN1, 13-bp deletion for TALEN2, and 18-bp insertion plus 7-bp deletion for TALEN3) (Fig. 1h). We further crossed these heterozygous animals (for TALEN1, females carrying the 19-bp deletion and males carrying the 1-bp deletion were used) to examine if the mutations affected embryonic development. Unexpectedly, we observed no LR or other defects in embryos derived from crosses between animals carrying mutations at the TALEN1 site (Fig. 2a-b'). Genotyping of 12 randomly selected 3-gill slit larvae (L3 stage) from the cross-identified 4 homozygous and 8 heterozygous/WT (wild type) individuals (Additional file 1: Fig. S3). The homozygous mutants can normally survive to adulthood. In contrast, we observed around 25\% (136/542 for the TALEN2 site and 141/550 for the TALEN3 site) of embryos showing specific and identical phenotypes in the anterior pharyngeal region at L3 stage, from crosses of animals with mutations at the TALEN2 or TALEN3 site. Genotyping analysis confirmed that all individuals showing these phenotypes were homozygous mutants, while individuals showing normal morphology were either Pitx heterozygotes or wild type (Additional file 1: Fig. S4, S5). Homozygous Pitx TALEN2 or TALEN3 mutants died at L4 stage. Compared to WT/heterozygotes larvae (Fig. 2c, c', e, e'), homozygous Pitx TALEN2 or TALEN3 mutants lacked a mouth opening, preoral pit, and CSG duct on the left side, and developed an endostyle and the glandular region of the CSG on each side (Fig. 2d, d', f, f'). The gill slits, which first opened at the ventral midline and then extended up to the right side of the head in wild type larvae (Fig. 2c', e'), were also affected in the two Pitx mutants: they appeared at the ventral side but failed to open and extended up to the right side (Fig. 2d', f'). Notably, these phenotypes were very similar to that observed in amphioxus larvae lacking the Nodal signaling pathway activity [28]. Since Pitx TALEN2 and TALEN3 mutants showed identical phenotype, Pitx TALEN2 mutants were used for further analysis. To simplify wording, we refer to Pitx TALEN2 mutants as Pitx mutants and those carrying mutations at the TALEN1 site as Pitxc mutants hereafter.

\section{Pitx mutants lack left-side organs but form incomplete right-side organs on the left side}

The finding that Pitx mutants in amphioxus showed a symmetrical pharyngeal phenotype as observed in embryos deficient of the Nodal signaling pathway is unexpected, as Pitx is generally thought as a determinant of left-side development but not a LR patterning gene. To verify this, we examined expression of marker genes of pharyngeal organs including the preoral pit, mouth, CSG, and endostyle in N5 and T1 embryos. In WT and Pitx ${ }^{+/}$embryos, Lhx3, Prop1, and Pit were expressed in the left anterior endoderm where the preoral pit will later form (Fig. 3a-f, arrowheads). Consistent with loss of the preoral pit in Pitx ${ }^{-/-}$larvae, expression of these genes in the prospective preoral pit region were lost in Pitx $^{-1-}$ embryos (Fig. 3a'-f'). Likewise, Pou4, which is normally expressed in the developing mouth on the left side (Fig. 3g, arrow), also lost its expression in Pitx ${ }^{-/-}$ mutants (Fig. 3g'). $N k x 2.1$ and Hex which are expressed in the endostyle are asymmetrically expressed in WT and Pitx ${ }^{+/-}$embryos in a $\mathrm{L}<\mathrm{R}$ manner (Fig. 3h-k). In Pitx $^{-1-}$ mutants, Nkx2.1 and Hex expression became symmetric, with the expression pattern on both sides being similar to that in the right side of wild type embryos (Fig. 3h'-k'). However, careful examination revealed that Nkx2.1 expression on the right side (Fig. 3i', black arrowhead) was not identical to its expression on the left side (Fig. 3i', red purple arrowhead): the former was extended more posteriorly than the latter (Fig. 3i'). This indicated that the endostyle region was almost but not fully duplicated on the left side of Pitx ${ }^{-1-}$ mutants. A similar case was also observed in the CSG of Pitx ${ }^{-1-}$ mutants. In WT and Pitx ${ }^{+-}$embryos, FoxE4 was expressed in the whole CSG including the right-sided glandular region and the left-sided duct (Fig. 3l, m), while Krox was transcribed only in the glandular region (Fig. 3n, o). In Pitx ${ }^{-/-}$embryos, although FoxE4 expression on the right side seemed to be fully duplicated on the left side (Fig. 3l', $\left.\mathrm{m}^{\prime}\right)$, Krox expression was not since its expression domain on the left side was slightly smaller than that on the right side (Fig. 3n', o'). These results demonstrated that the pharyngeal structure of Pitx ${ }^{-1-}$ mutants was not fully symmetrical unlike that observed in embryos lacking Nodal signaling pathway [28]. Consistent with this, asymmetric expression patterns of the LR patterning genes, like Dand5, Nodal, Lefty, and Pitx were not altered in Pitx ${ }^{-/}$embryos at N0 (Additional file 1: Fig. S6a'), N2 (Additional file 1: Fig. S6b', c', d'), N4 (Additional file 1: Fig. S6e', f', g'), or T1 stages (Additional file 1: Fig. S6h', i', j'), although Pitx expression was slightly 


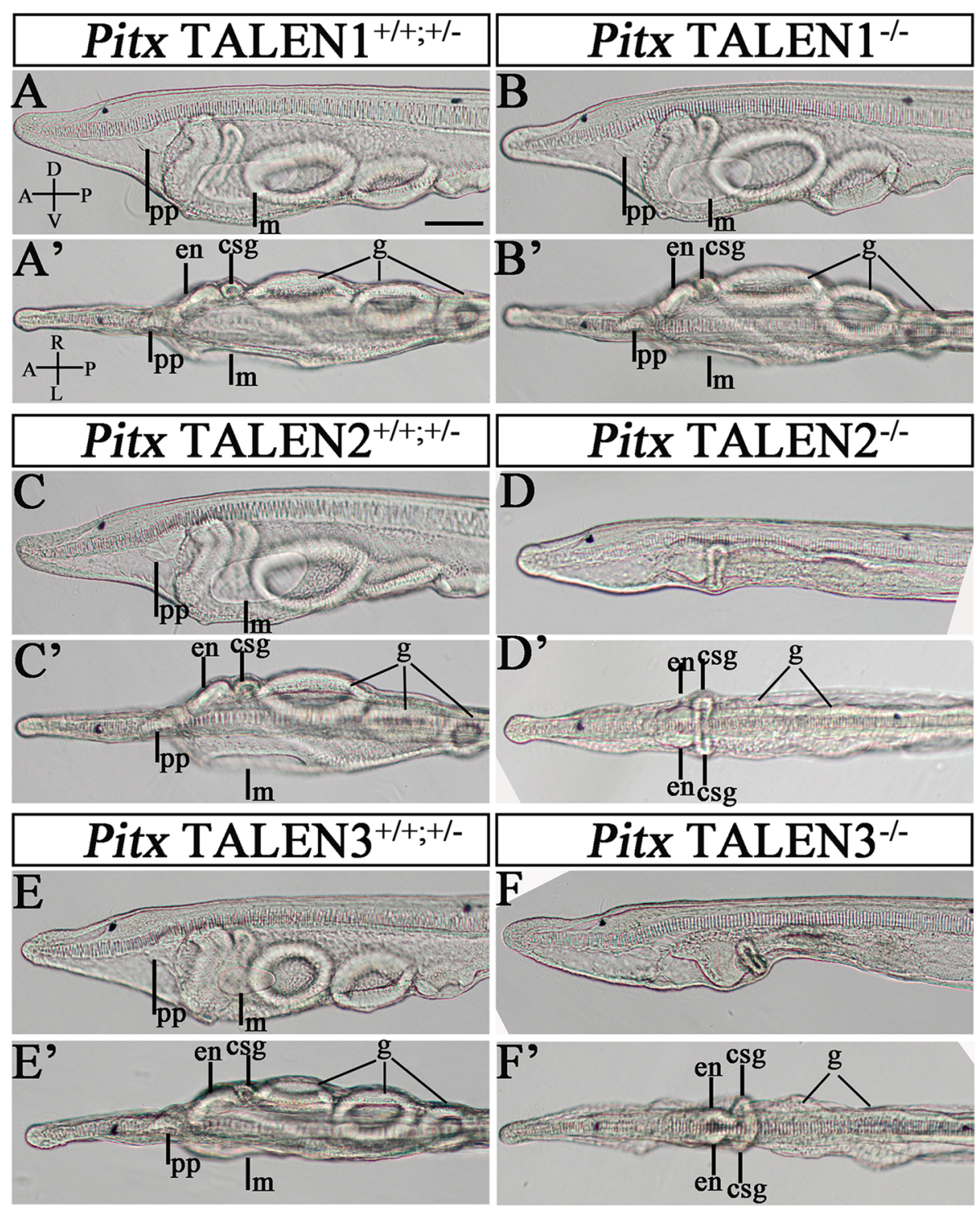

Fig. 2 Pitx mutants phenotype. In Pitx TALEN1 $1^{+/+;+-}$larvae (a, a'), Pitx TALEN2 ${ }^{+/+;+/-}$larvae $\left(\mathbf{c}, \mathbf{c}^{\prime}\right)$, and Pitx TALEN3 ${ }^{+/+;+/-}$larvae $\left(\mathbf{e}, \mathbf{e}^{\prime}\right)$, the preoral pit and mouth are formed on the left side, with the endostyle, club-shaped gland (CSG) and first gill slit developing on the right side. Pitx TALEN $1^{-/-}$mutants show normal phenotype $\left(\mathbf{b}, \mathbf{b}^{\prime}\right)$. Pitx TALEN2 ${ }^{-1-}$ mutants $\left(\mathbf{d}, \mathbf{d}^{\prime}\right)$ and Pitx TALEN3 ${ }^{-/-}$mutants $\left(\mathbf{f}\right.$, $\left.\mathbf{f}^{\prime}\right)$ lack a mouth opening and preoral pit on the left side, forming an endostyle and CSG on each side. The larvae at L3 (3 gill slits larva) stage are observed in lateral views in (a $\mathbf{b}, \mathbf{c}, \mathbf{d}, \mathbf{e}, \mathbf{f})$ and dorsal views in $\left(\mathbf{a}^{\prime}, \mathbf{b}^{\prime}, \mathbf{c}^{\prime}, \mathbf{e}^{\prime}, \mathbf{f}^{\prime}\right)$. m, mouth; pp: preoral pit; en, endostyle; csg, club-shaped gland; g: gill slit. A, anterior; P, posterior; $D$, dorsal; $V$, ventral; $L$, left side; R; right side. Scale bars:50 $\mu \mathrm{m}$

downregulated in Pitx ${ }^{-/-}$embryos at all examined stages (Additional file 1: Fig. S6d', g', j') and Nodal, Lefty, and Pitx expression in the forming preoral pit was lost at T1 stage (Additional file 1: Fig. S6h', i', j').

In addition to the LR organs examined above, Hatschek's nephridium, hematopoietic domains, and Hatschek's right diverticulum (short for HRD) are also positioned asymmetrically on one side of amphioxus embryos. Among them, the HRD is derived from the right anterior-dorsal endoderm, and Hatschek's nephridium and hematopoietic domains are developed from the left first and right first somites, respectively [38, 39]. To evaluate if the development or positioning of these organs were disrupted due to Pitx loss-offunction, we analyzed genes specifically expressed in them in Pitx ${ }^{-/}$mutants at N5 stage. In WT and Pitx ${ }^{+/-}$embryos, Gata1/2/3, Pdvegfr, and Scl exhibited two bilateral and slightly asymmetrical expression domains, which respectively denoted left-side Hatschek's nephridium (Fig. 4c, e, g, blue arrowhead) and the right-side hematopoietic domain (Fig. 4c, e, g, black arrowhead) [38]. In Pitx ${ }^{-/-}$mutant embryos, however, their expression was bilaterally symmetric (Fig. 4d, $\mathrm{f}$, h, black arrowhead). To determine the identity of the 


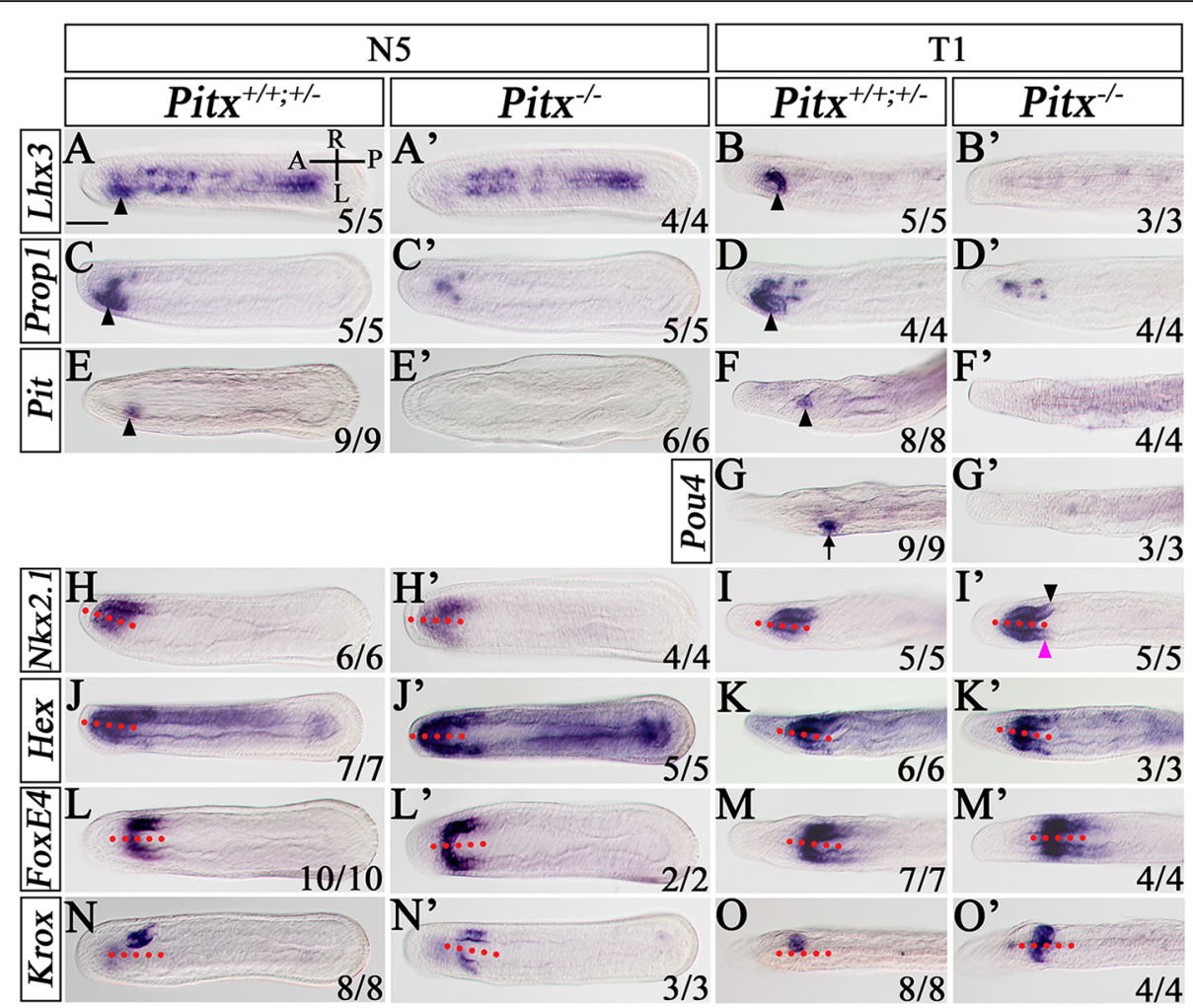

Fig. 3 Pharyngeal organ-specific gene expression in Pitx TALEN2 $2^{-/}$mutants. In situ hybridization to Pitx mutants at N5 and T1 stage for Lhx3 (a, $\left.\mathbf{a}^{\prime}, \mathbf{b}, \mathbf{b}^{\prime}\right)$, Prop $1\left(\mathbf{c}, \mathbf{c}^{\prime}, \mathbf{d}, \mathbf{d}^{\prime}\right)$, and Pit $\left(\mathbf{e}, \mathbf{e}^{\prime}, \mathbf{f}, \mathbf{f}^{\prime}\right) . \mathbf{g}, \mathbf{g}^{\prime}$ In situ hybridization to Pitx mutants at T1 stage for Pou4. Arrowheads indicate the preoral pit region, arrows indicate the mouth region. In situ hybridization to Pitx mutants at N5 and T1 stage for Nkx2.1 (h, h', $\left.\mathbf{i}, \mathbf{i}^{\prime}\right)$, Hex (j, $\left.\mathbf{j}^{\prime}, \mathbf{k}, \mathbf{k}^{\prime}\right)$, FoxE4 (I, I', $\left.\mathbf{m}, \mathbf{m}^{\prime}\right)$, and $\operatorname{Krox}\left(\mathbf{n}, \mathbf{n}^{\prime}, \mathbf{o}, \mathbf{o}^{\prime}\right)$. In $\mathbf{i}^{\prime}$, the black arrowhead shows the expression boundary of Nkx2.1 on the right side and red purple arrowhead shows expressing boundary on the left side. All images are dorsal views. Numbers in the bottom right corner of a panel show the number of times the phenotype depicted was observed, out of the total number of embryos from that genotype analyzed. A, anterior; P, posterior; L, left side; R; right side. Scale bars: $50 \mu \mathrm{m}$

expression domains, we analyzed $\operatorname{Pax} 2 / 5 / 8$ gene, which is expressed in Hatschek's nephridium but not in the hematopoietic center [40]. We found that Pax 2/5/8 expression in the prospective nephridium is absent in Pitx ${ }^{-/-}$mutants (Additional file 1: Fig. S7c, d). This result indicated that Pitx ${ }^{-/}$mutants lacked Hatschek's nephridium but formed a hematopoietic domain on each side. We also noticed a Gata1/2/3 expression domain in the right-side HRD in normal embryos (Fig. 4c, arrow) and found that this domain was present on both sides of Pitx ${ }^{-/-}$mutants (Fig. 4d, arrow). Moreover, asymmetric expression $(\mathrm{R}>\mathrm{L})$ of the Hand gene in the lateral/ventral mesoderm derived from amphioxus somites [38] became bilaterally expressed in Pitx ${ }^{-/-}$mutants although still in a $\mathrm{R}>\mathrm{L}$ pattern (Fig. 4b).

Together, the above results indicate that in Pitx mutants, all left-side organs are lost while the rightside organs form normally on the right side and ectopically (albeit not incompletely) on the left side.
Pitx acts downstream of Nodal signaling to control asymmetric arrangement of amphioxus somites and peripheral nerves

Different from vertebrates, amphioxus somites and peripheral nerves (aligned with the somites) are asymmetrically arranged along the LR axis, with the left ones being offset about half a segment anterior to the right ones [41]. These asymmetries become first recognizable at mid-neurula (with 8-10 somites) when somitogenesis shifts from a symmetrical way by enterocoelous outpocketing of paraxial mesoderm, to a left-to-right alternate fashion by pinching off from the tailbud [42]. To assay if the arrangement of the somites and peripheral nerves were affected in Pitx ${ }^{-/}$mutants, we examined expression of the m-actin gene, a marker of the somites at stage N5, and acetylated tubulin, a marker of the peripheral nerves at L3 stage [28]. We found both markers were asymmetrically expressed in the mutants, similar to that observed in WT and heterozygous embryos (Fig. 4i, k). However, compared to WT and heterozygous 


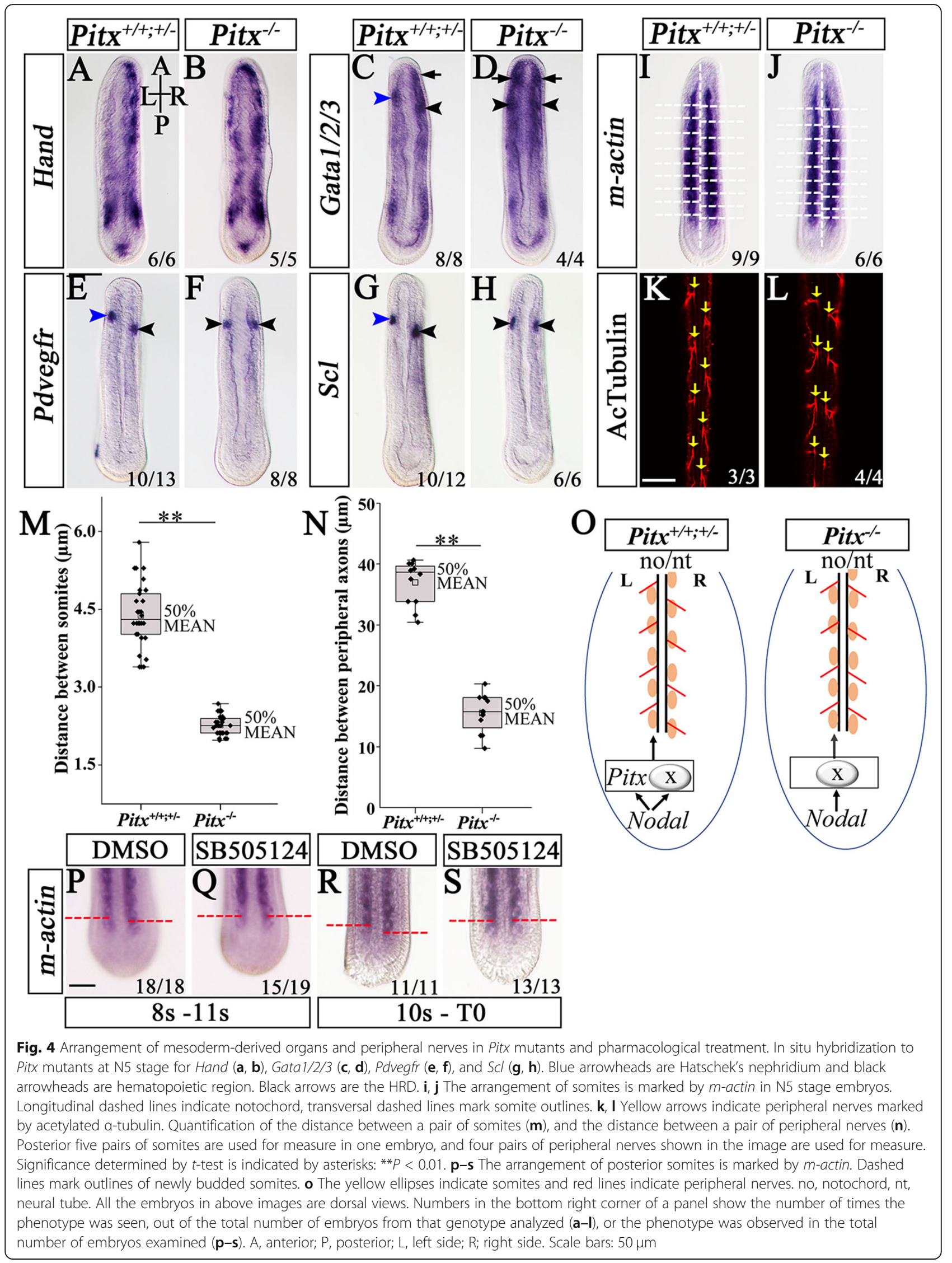


embryos, the mutants exhibited significantly shorter distances between the left somites or peripheral nerves and their corresponding ones on the right side (Fig. 4j, l, m, $\mathrm{n})$. These results showed that the asymmetric arrangement of the somites and peripheral nerves was weakened but not abolished in Pitx ${ }^{-/-}$mutants.

Between N5 and early larvae stages, both Nodal and Pitx are constantly expressed in the left side of the amphioxus tailbud (Additional file 1: Fig. S6e, g, h, j, arrowheads). We speculated that Nodal signaling pathway acts upstream of Pitx to determine somite asymmetry. To test this, we treated embryos with the Nodal signaling inhibitor SB505124 in different developmental windows and examined Pitx expression as well as the asymmetry of newly formed somites. To exclude the influence of Nodal function in early LR patterning on the analysis, we treated embryos with the drug after the N4 stage when the LR axis is thought to have been fixed $[28,33]$, namely from $8 \mathrm{~S}$ (neurula with 8 somites) to $11 \mathrm{~S}$ (neurula with 11 somites), and from $10 \mathrm{~S}$ (neurula with 10 somites) to around T0. As expected, both treatments eliminated Pitx expression in the tailbud of the treated embryos (Additional file 1: Fig. S8a'-d') and abolished the asymmetry of the newly budded somites (Fig. 4p-s). These results indicated that the Nodal signaling pathway regulated the asymmetric generation of somites from the tailbud, and that besides Pitx, other factors (X) downstream of Nodal signal also played vital roles in the process (Fig. 4o). We also conducted treatments on later developmental stages, from $\mathrm{T} 0$ to $\mathrm{T} 1$, and from $\mathrm{T} 1$ to $\mathrm{L} 0$ stage. Similarly, both treatments could effectively eliminate Pitx expression from the tailbud (Additional file 1: Fig. S8i'-1'). We did not assess if the treated embryos had symmetric somites, since somites at these stages of embryos were not easy to recognize.

\section{Pitx is required for amphioxus LR organ development in a dosage-sensitive manner}

We also crossed heterozygous Pitxc animals (carrying mutation at the TALEN1 site) with heterozygous Pitx animals carrying mutations at the TALEN2 site or the TALEN3 site. Unexpectedly, in both crosses, we found around a quarter of the generated embryos showing a milder (compared to Pitx $^{-1-}$ mutants), but specific phenotype at stage L3. Genotypic analysis confirmed that individuals showing this phenotype carry mutations at both TALEN1 site and TALEN2 or TALEN3 site TALEN1 $^{+/-}$; TALEN2 ${ }^{+/-}$or TALEN1 $^{+/-}$; TALEN $^{+/-}$), while others of normal morphology harbor mutations only in one or in neither of the TALEN1 site and the TALEN2 or TALEN3 site $\left(\right.$ TALEN1 $^{+/+}$; TALEN2 ${ }^{+/+}$, TALEN $^{+/-} ; \quad$ TALEN2 $^{+/+}, \quad$ TALEN $^{+/+} ; \quad$ TALEN2 $^{+/-}$, TALEN $^{+/+} ;$TALEN $^{+/+}$, TALEN $^{+/-} ;$TALEN3 $^{+/+}$, or TALEN $^{+/+}$; TALEN $^{+/-}$) (Additional file 1: Fig. S9, S10). For simplification, we hereafter refer to these two types of embryos as Pitxa/c homozygotes and WT/heterozygotes respectively and use embryos from crosses between TALEN1 and TALEN2 animals for further analysis. Compared to WT/heterozygotes (Fig. 5a, a'), Pitxa/c homozygotes had no mouth opening and malformed preoral pit but formed an endostyle and glandular region of the CSG on each side (Fig. $5 \mathrm{~b}, \mathrm{~b}$ '). Importantly, their gill slits still formed and positioned normally as that of WT/heterozygotes (Fig. 5a', b').

To further clarify the phenotype of Pitxa/c homozygotes, we examined expression of marker genes of LR organs in the mutants at N5 and T1 stages. Pitxa/c homozygotes lacked Pou4 expression (marker of the mouth) on the left side (Fig. 5i, i', arrow), but expressed $N k \times 2.1$ (Fig. 5j, k) and Hex (Fig. 5l, m) (markers of the endostyle), and FoxE4 (Fig. 5n, o) and Krox (Fig. 5p, q) (markers of the CSG) nearly symmetrically on both sides (Fig. 5j'-q') as observed in Pitx ${ }^{-1-}$ mutants (Fig. 3h'-o'). Interestingly, Lhx3, Prop1, and Pit expression in the posterior preoral pit region (Fig. $5 \mathrm{c}-\mathrm{h}$, red arrowhead) was lost in Pitxa/c homozygotes (Fig. 5c'-h') as in Pitx ${ }^{-/-}$ mutants (Fig. 3a'-f'), while that of $L h x 3$ and Prop 1 in the anterior part of the organ remained in the former (Fig. $5 c^{\prime}-f^{\prime}$, black arrowhead) but was lost in the latter (Fig. $\left.3 a^{\prime}-d^{\prime}\right)$. The asymmetric expression of Hand in the lateral/ventral mesoderm (Fig. 6a, a'), Gata1/2/3, Pdvegfr, and $\mathrm{Scl}$ in Hatschek's nephridium (Fig. 6b, d, e, blue arrowhead) and hematopoietic domain (Fig. 6b, d, e, black arrowhead), and Gata1/2/3 in the HBD (Fig. 6b, arrow) were not affected in Pitxa/c mutants (Fig. 6b', d', $\mathrm{e}^{\prime}$ ), while that of m-actin in the somites (Fig. 6c, c', g) and acetylated tubulin in the peripheral nerves (Fig. 6f, $\mathrm{f}^{\prime}, \mathrm{h}$ ) was weakened as observed in Pitx ${ }^{-/-}$mutants (Fig. $4 \mathrm{i}-\mathrm{n})$.

Together, the above results indicated that the mutation at the TALEN1 site actually affected Pitxc function, albeit very mildly, and that the development of various LR organs are of different sensibility to Pitx activity reduction. Among them, the mouth, endostyle, CSG, posterior part of the preoral pit, and the asymmetry of the somites and peripheral nerves were sensitive to an intermediate level of Pitx activity reduction (Pitxa/c homozygotes), while the lateral/ventral mesoderm, Hatschek's nephridium, hematopoietic domain, HBD, and the anterior part of the preoral pit were sensitive to total loss of Pitx activity (Pitx $\left.{ }^{--}\right)$, with none of them sensitive to a low level of Pitx activity reduction $\left(\right.$ Pitxc $\left.^{-/-}\right)$.

\section{Pitx controls development of pharyngeal LR asymmetric organs by directly activating genes essential for left-side organs and repressing genes of right-side organs on the left side}

Among LR asymmetric organs within the amphioxus pharynx, the preoral pit and endostyle are thought to be homologs of the vertebrate pituitary and thyroid 

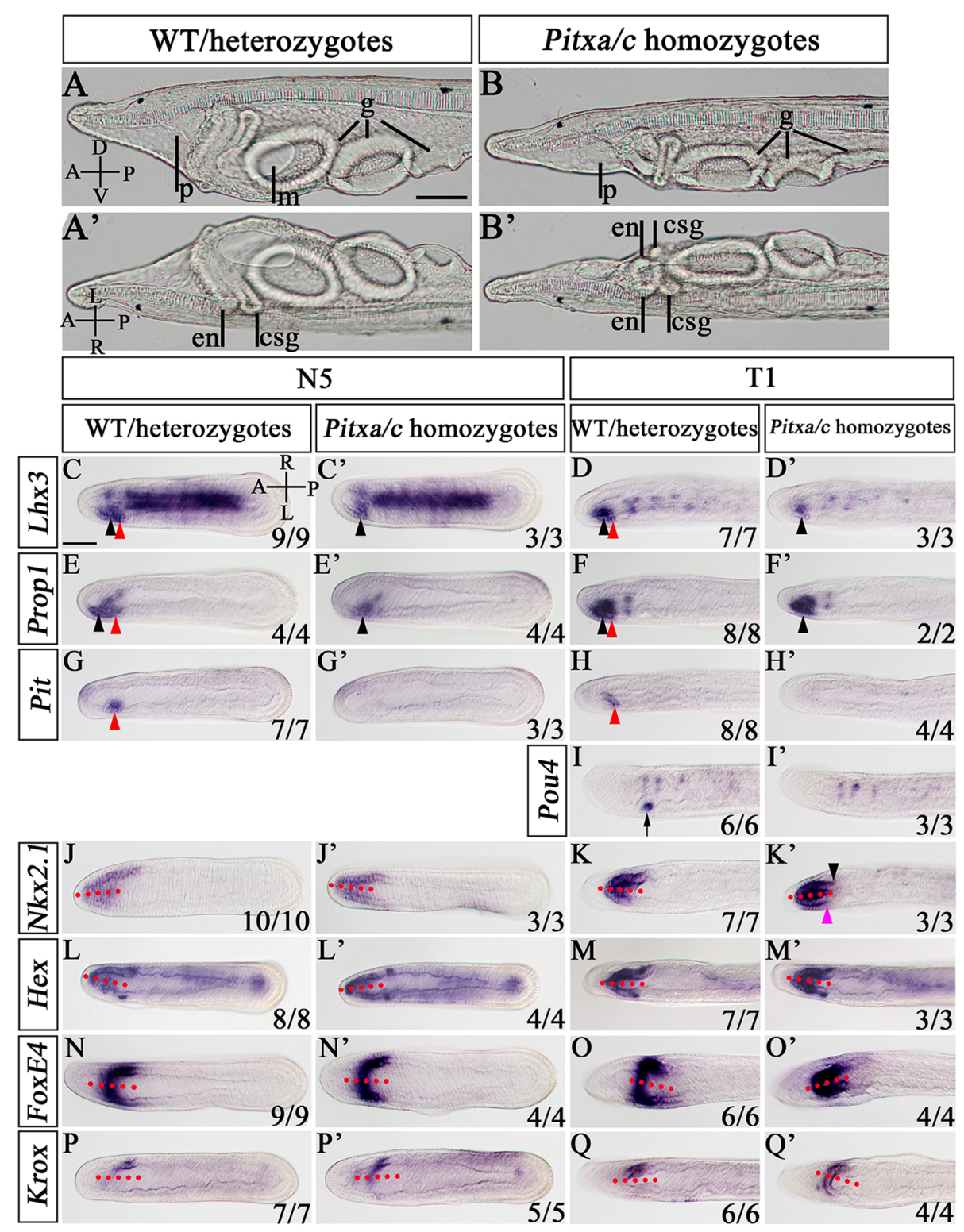

Fig. 5 Partial loss of Pitx function exhibits a mild phenotype and disturbs phanyngeal organs asymmetry. $\mathbf{a}, \mathbf{b}$ Left lateral view of the $L 3$ larvae (3-gill slits). $\mathbf{a}^{\prime}, \mathbf{b}^{\prime}$ The lavae are shown in a view between right lateral and ventral view, which clearly shows that the endostyle and CSG are symmetrical in Pitxa/c homozygotes (b'). m, mouth; pp: preoral pit; en, endostyle; csg, club-shaped gland; g: gill slit. In situ hybridization to WT/heterozygotes and Pitxa/c homozygotes at N5 and T1 stage for Lhx3 (c, $\left.\mathbf{c}^{\prime}, \mathbf{d}, \mathbf{d}^{\prime}\right)$, Propl (e, e', $\left.\mathbf{f}, \mathbf{f}^{\prime}\right)$, and Pit $\left(\mathbf{g}, \mathbf{g}^{\prime}, \mathbf{h}, \mathbf{h}^{\prime}\right.$ ). i, $\mathbf{i}^{\prime}$ In situ hybridization to WT/heterozygotes and Pitxa/c homozygotes at T1 stage for Pou4. Black arrowheads indicate the anterior preoral pit region and red arrowheads indicate the posterior preoral pit region, arrows indicate the mouth region. In situ

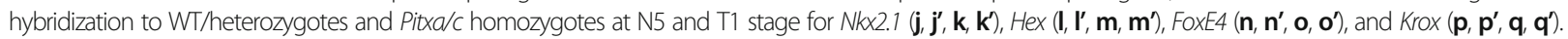
In ( $\left.\mathbf{k}^{\prime}\right)$, the black arrowhead shows the expression boundary of Nkx2.1 on the right side and red purple arrowhead shows expressing boundary on the left side. Images are dorsal views in $\mathbf{c}-\mathbf{q}^{\prime}$. Numbers in the bottom right corner of a panel show the number of times the phenotype depicted was observed, out of the total number of embryos from that genotype analyzed. A, anterior; P, posterior; D, dorsal; V, ventral; L, left side; R; right side. Scale bars: $50 \mu m$

respectively [43, 44], while the CSG is an amphioxusspecific organ transiently present at larval stages. Lhx3Prop1-Pit and Hex-Nkx2.1 are required for the development of pituitary $[43,45,46]$ and thyroid in vertebrates respectively [44, 47], and FoxE4 is one of the earliest transcription factors activated specifically in the CSG [48]. To dissect how Pitx regulated development of the preoral pit, endostyle, and CSG in amphioxus, we examined the expressions of these genes and compared them with that of Pitx in embryos between N1 stage, when Pitx begins to express asymmetrically on the left side [24], and N4 stage, when LR morphogenesis is thought to initiate [28]. At the N1 stage, Pitx was detected in the left dorso-anterior half of the embryos (Fig. 7a1, a1"'), 


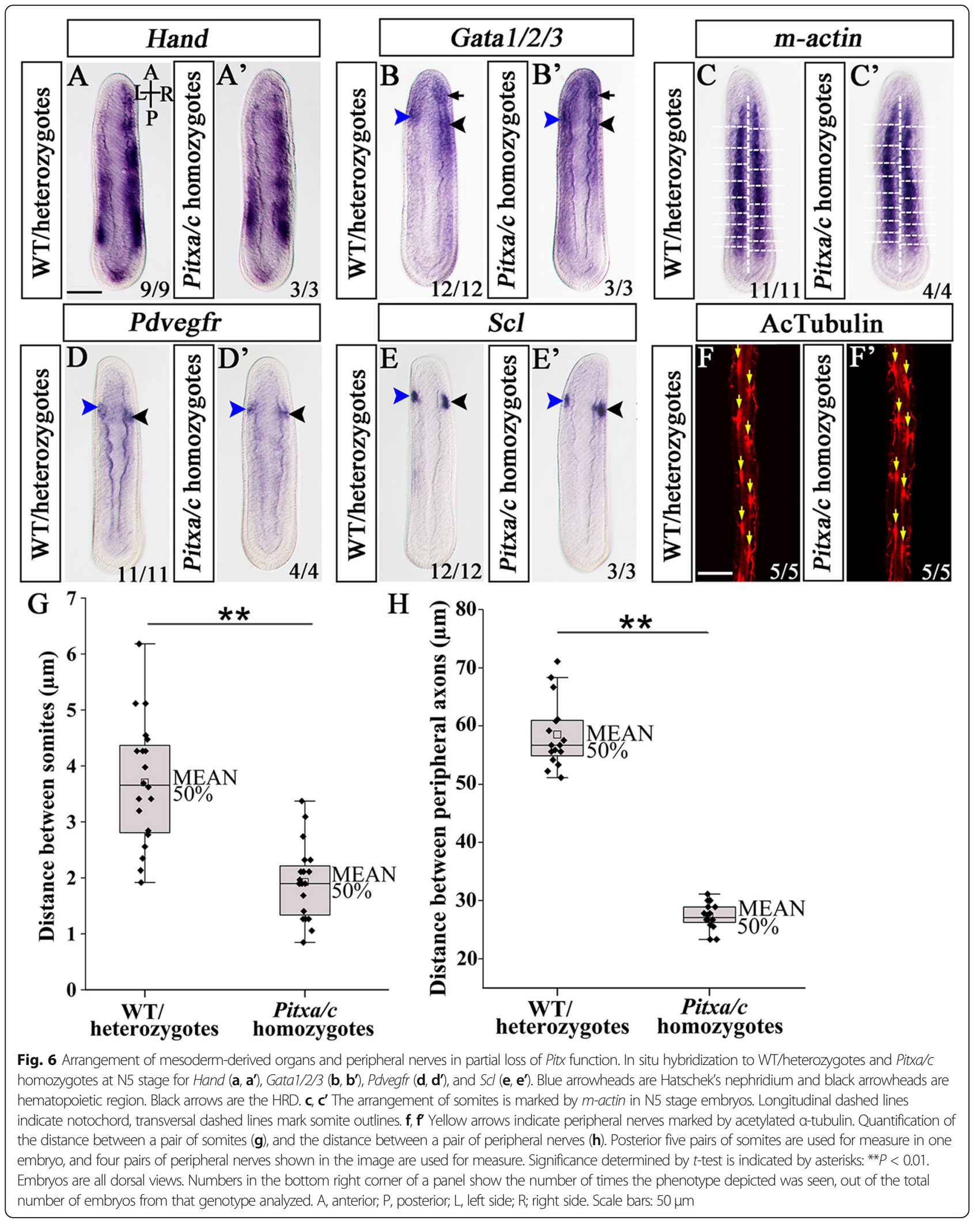




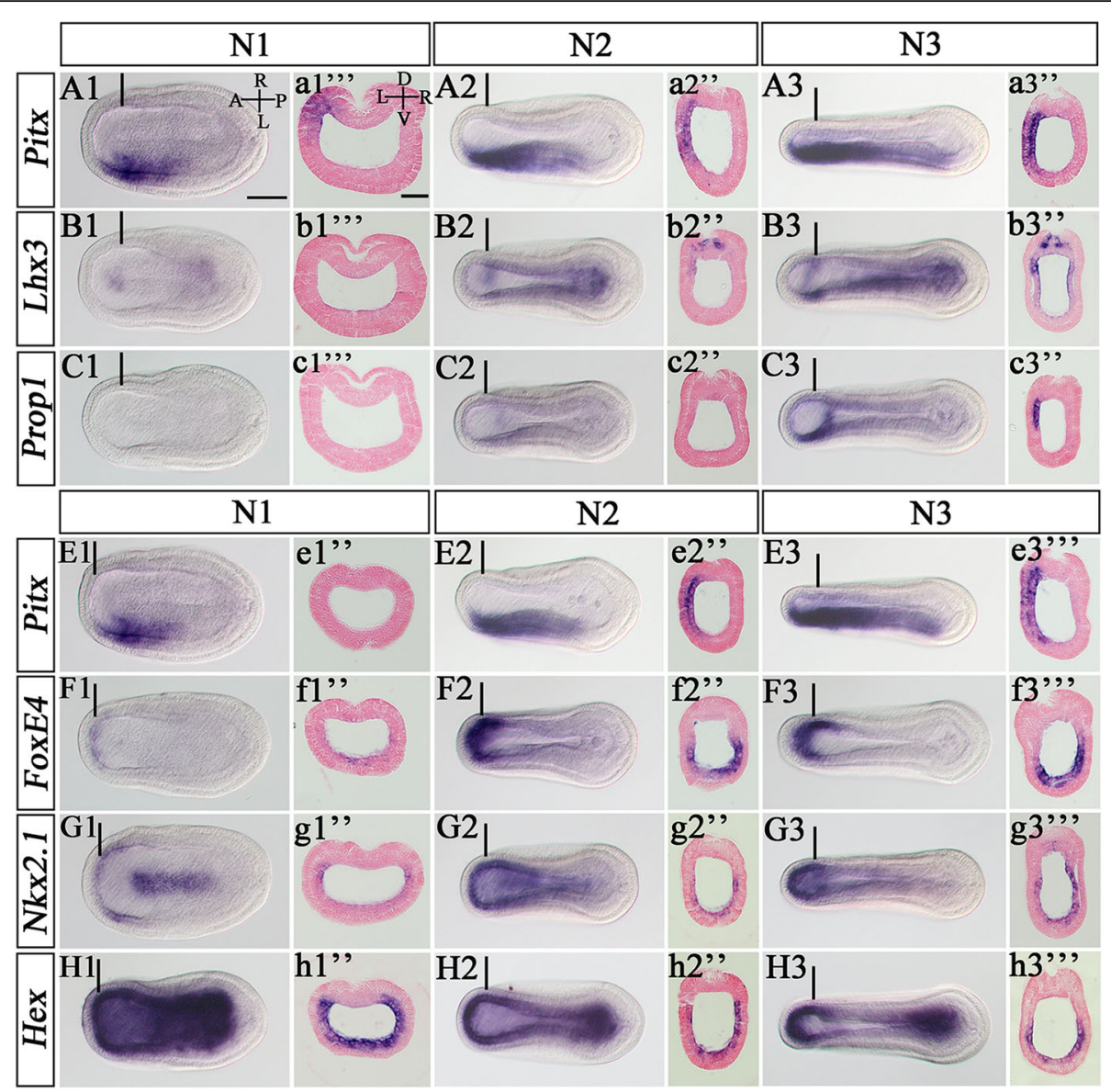

Fig. 7 Expression pattern of Pitx and asymmetrical pharyngeal organs markers. a1-c3, e1-e3 Pitx is expressed in the left anterior-dorsal half of the embryos from the $\mathrm{N} 1$ neurula stage to N3 neurula stage (a1-a3, e1-e3), Lhx3, Prop1 is activated in the prospective preoral pit region from the N1 stage to N3 stage (b1-b3, c1-c3), as shown by WISH and transvers sections in (a1"'--c3"). $\mathbf{f 1 - h} 3$ The expression of right-sided organ makers, FoxE4 (f1-f3), Nkx2.1 (g1-g3), and Hex (h1-h3) from N1 to N3 stage, as shown by WISH and transverse sections (f1"'-h3"'). The lines in a1-h3 indicate the position of transverse sections of the embryos. The embryos detected by WISH are all in dorsal views. A, anterior; P, posterior; D, dorsal; $V$, ventral; $L$, left side; R; right side. Scale bars: $50 \mu \mathrm{m}$

while Lhx3 was weakly and Prop1 was not yet activated in the left anterior endoderm that will later become preoral pit (Fig. 7b1, b1"', c1, c1"'). At this stage, FoxE4 began to be transcribed in the anterior most endoderm (Fig. 7f, f1") where Pitx is not expressed (Fig. 7e1,e1"). $N k \times 2.1$ and Hex expression in the anterior mesendoderm, from which the endostyle will later form, are symmetrically activated from the early neurula stage [44, 47]. However, at the N1 stage, the expression levels of both genes in the left anterior paraxial mesoderm were somewhat lower than that in the right paraxial mesoderm (Additional file 1: Fig. S11g1, g1"', h1and h1"'). At N2 stage, Pitx expression was enhanced and expanded both anteriorly, posteriorly and ventrally, forming a gradient along these axes (Fig. 7a2, a2", e2 and e2", Additional file 1: Fig. S11a2'-a2"', e2'-e2"'). Along with this, Lhx3 and Prop1 were both transcribed in the region where the preoral pit will later form (Fig. 7b2, b2", c2 and c2"), and FoxE4, Nkx2.1, and Hex were further downregulated on the left side (Fig. 7f2, f2", g2, g2", h2 and h2"). Interestingly, FoxE4, Nkx2.1, and Hex also showed a gradient of expression on the left side along the dorsal-ventral and anterior-posterior axis (Additional file 1: Fig. S11f2'-f2"', g2'-g2"', h2'-h2"'), which were inversely correlated to the gradient of Pitx expression (Additional file 1: Fig. S11e2'-e2"'). By N3 stage, Pitx expanded its expression domain more ventrally and posteriorly (Fig. 7a3, a3", e3, and e3"', Additional file 1: Fig. S11a3'-a3"', e3'-e3"'), and the asymmetric expression of Lhx3 and Prop1 on the left side (Fig. 7b3, b3", c3, c3", Additional file 1: Fig. S11b3'-b3"', e3'-e3"') and that of FoxE4, Nkx2.1, and Hex on the right side became more pronounced (Fig. 7f3"', g3"' , h3"', Additional file 1: Fig. S11f3'-f3"', g3'-g3"' , h3'-h3"'). Compared to those genes, Pit expression in the posterior part of the preoral pit and Krox expression in the endostyle were activated 
relatively late and became detectable until $\mathrm{N} 4$ stage (Additional file 1: Fig. S12).

The activation of $L h x 3$ and Prop 1 and downregulation of FoxE4, Nkx2.1 and Hex in areas of Pitx expression suggests that Pitx could induce expression of the former while inhibiting expression of the latter. To test this, we first examined the expression of these genes in Pitx ${ }^{-/-}$ mutants at N2 and N3 neurula stages (Additional file 1: Fig. S13). As described above, FoxE4, Nkx2.1, and Hex were asymmetrically expressed in the anterior endoderm of WT and Pitx ${ }^{+/-}$embryos in an apparently $\mathrm{L}<\mathrm{R}$ manner at the N2 stage (Additional file 1: Fig. S13a, a'-a"', c, $c^{\prime}-c^{\prime \prime}$, e, e'-e"'). However, in Pitx ${ }^{-/-}$mutants the asymmetric expression pattern of these genes was somewhat weakened and became less pronounced due to an upregulation of their expression on the left side (Additional file 1: Fig. S13b, b'-b"', d, d'-d"', f, f'-f"'). At the N3 stage, asymmetry of FoxE4, Nkx2.1, and Hex expression became more pronounced in WT and Pitx ${ }^{+-}$embryos (Additional file 1: Fig. S13g, g'-g"', i, i'-i'", k, k'-k"'), but were still ambiguous in Pitx ${ }^{-/-}$mutants due to a failure of downregulation on the left side (Additional file 1: Fig. S13h, h'-h"', j, j'-j"', i, i'-i"'). At this stage, both $L h x 3$ and Prop1 expression were not activated in the presumptive preoral pit of the Pitx ${ }^{-1-}$ mutants (Additional file 1: Fig. S13m-p). To further clarify the speculation above, we injected unfertilized amphioxus eggs with Pitxc mRNA and examined its effect on the expression of FoxE4, $N k x 2.1$, and $L h x 3$ genes. FoxE4 was expressed specifically in the anterior endoderm of all (11/11) examined uninjected embryos (Fig. 8a). However, after injection of Pitxc mRNA, 43.8\% (7/16) of embryos showed no FoxE4 expression in the corresponding region (Fig. 8a'). A similar case was observed for $N k x 2.1$ (Fig. 8c, c'). Different to these, injection caused $28.6 \%(4 / 14)$ of embryos to express $L h \times 3$ ectopically in the right anterior endoderm (Fig. 8e', red arrow). We also injected Pitxc mRNA into one blastomere at a 2-cell stage, divided them into leftside injected and right-side injected ones at late gastrula stage, and then assayed the expression of the above genes. Among left-side injected embryos, 33.3\% (3/9) and $42.9 \%(3 / 7)$ of them showed no FoxE4 and Nkx2.1

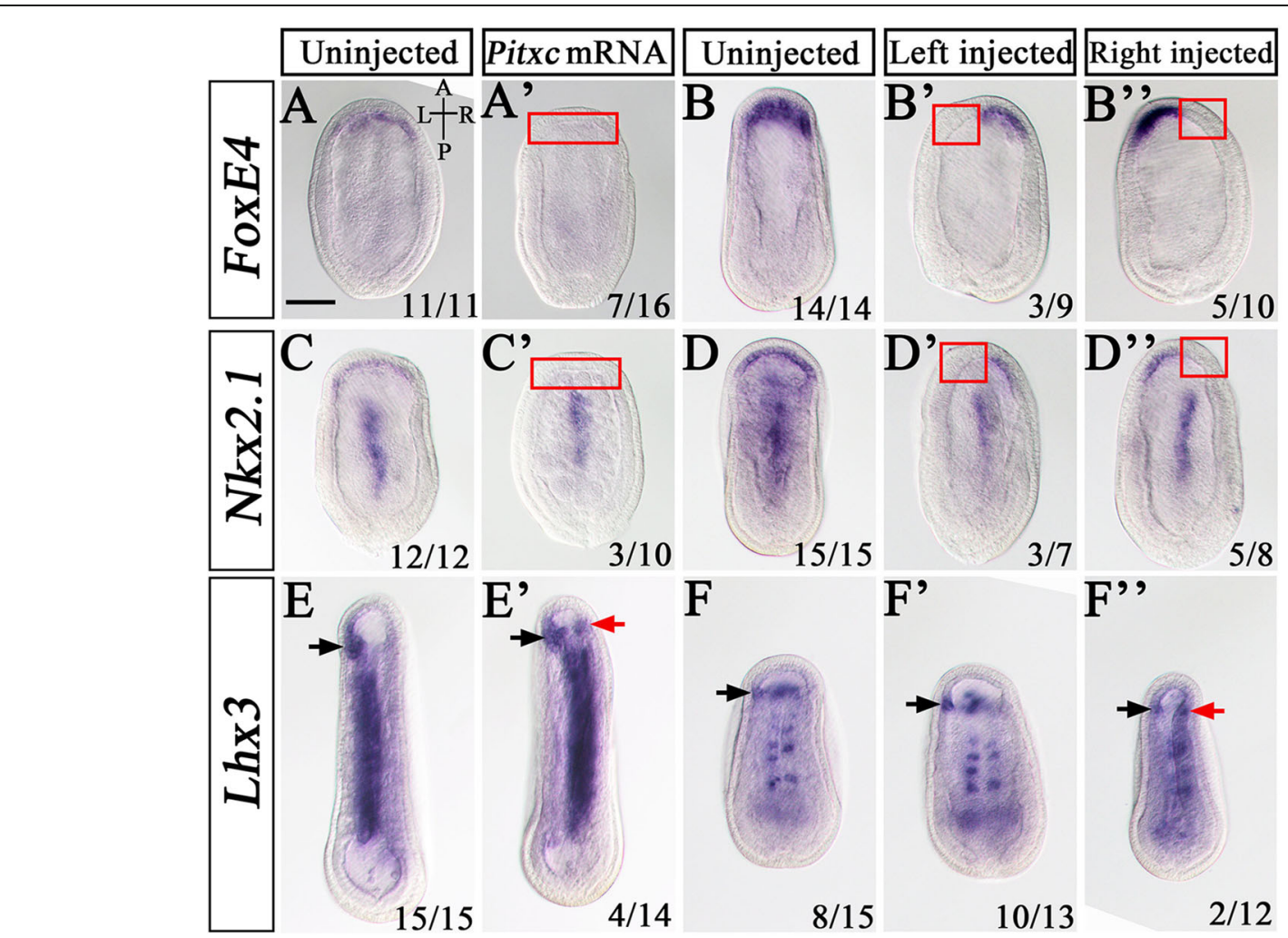

Fig. 8 Overexpression of Pitx can activate Lhx3 expression and inhibit the expression of FoxE4 and Nkx2.1. a-f These control groups are not injected with Pitxc mRNA. $\mathbf{a}^{\prime}-\mathbf{e}^{\prime}$ These groups are injected with Pitxc mRNA at the unfertilized egg stage. $\mathbf{b}^{\prime}-\mathbf{f}^{\prime \prime}$ Pitxc mRNA is injected into one blastomere at 2-cell stage. Red boxes indicate the region in which FoxE4 or Nkx2.1 expression disappeared. The black arrows indicate left preoral pit marked by Lhx3 and red arrows indicate the new induced preoral pit. The embryos detected is at N1 stage in (a, a', $\left.\mathbf{c}, \mathbf{c}^{\prime}\right)$, N2 stage in $\mathbf{b}-\mathbf{b}^{\prime \prime}, \mathbf{d}-\mathbf{d}^{\prime \prime}, \mathbf{f}-\mathbf{f}^{\prime \prime}$, N4 stage in $\mathbf{e}$, e'. Numbers in the bottom right comer of a panel show the number of times in phenotype was observed in total number of embryos examined. The remaining injected embryos not shown showed similar gene expression patterns like the uninjected embryos. All the images are dorsal view. A, anterior; $P$, posterior; $L$, left side; $R$; right side. Scale bars: $50 \mu \mathrm{m}$ 
expression in the left anterior endoderm, respectively (Fig. 8b, b', d, d'), while 76.9\% (10/13) exhibited Lhx3 expression in the left-side presumptive preoral pit region, which is significantly more than that $(53.3 \%, 8 / 15)$ observed in the uninjected embryos (Fig. 8f, f', arrow). In contrast, among right-side injected embryos, 50\% (5/10) and $62.5 \%(5 / 8)$ of them respectively lost FoxE4 and $N k x 2.1$ expression in the right anterior endoderm (Fig. 8b, b", d, d"), while $16.7 \%$ (2/12) showed ectopic $L h x 3$ expression in the right side (Fig. 8f, f", red arrow). Together, these results indicated that Pitx was required and sufficient for inducing $L h \times 3$ and Prop 1 expression and inhibiting FoxE4, Nkx2.1, and Hex expression in amphioxus embryos.

Lhx3 and Hex are upstream transcription factors for the development of vertebrate pituitary and thyroid, respectively $[49,50]$, and are among the earliest genes activated in the amphioxus preoral pit and endostyle, respectively $[45,47]$. We therefore speculated that Pitx regulated the development of the preoral pit and endostyle through directly regulating the expression of $L h x 3$ and Hex. To test this, we analyzed upstream sequences of the two genes and identified three Pitx binding sites for each gene (Fig. 9a, d, Additional file 1: Table S1). Luciferase assay showed that both sequences were able to drive significantly higher levels of luciferase activity than the control (Fig. 9b, c, e, f). Co-injection of Pitxc mRNA with the constructs increased luciferase activity of $L h \times 3$ construct (Fig. 9b), but decreased luciferase activity of Hex construct (Fig. 9e). Moreover, mutation of the Pitx binding sites abolished activity of $L h x 3$ sequence (Fig. 9c), but increased activity of Hex sequence (Fig. 9f). These results suggested that $L h x 3$ and Hex were likely direct transcriptional targets of Pitx in amphioxus, and Pitx binding activated Lhx3 expression, while Pitx binding repressed Hex expression.

\section{Discussion}

Requirement of Pitx2 for LR morphogenesis has been demonstrated in mice by gene knockout studies [7-10] and in chick and frogs using gene knockdown strategies [7-10]. However, its role in zebrafish LR morphogenesis is still under debate. Using a morpholino knockdown method, Liu and Semina found no LR defects in zebrafish Pitx2 morphants [16], while Garric et al. reported a weak phenotype in habenular asymmetry [17]. In a later study, Ji et al. confirmed the finding of Liu and Semina by examining several lines of Pitx 2 mutants and revealed that these mutants are able to survive to adults without any LR defects $[15,16]$. Outside vertebrates, Pitx

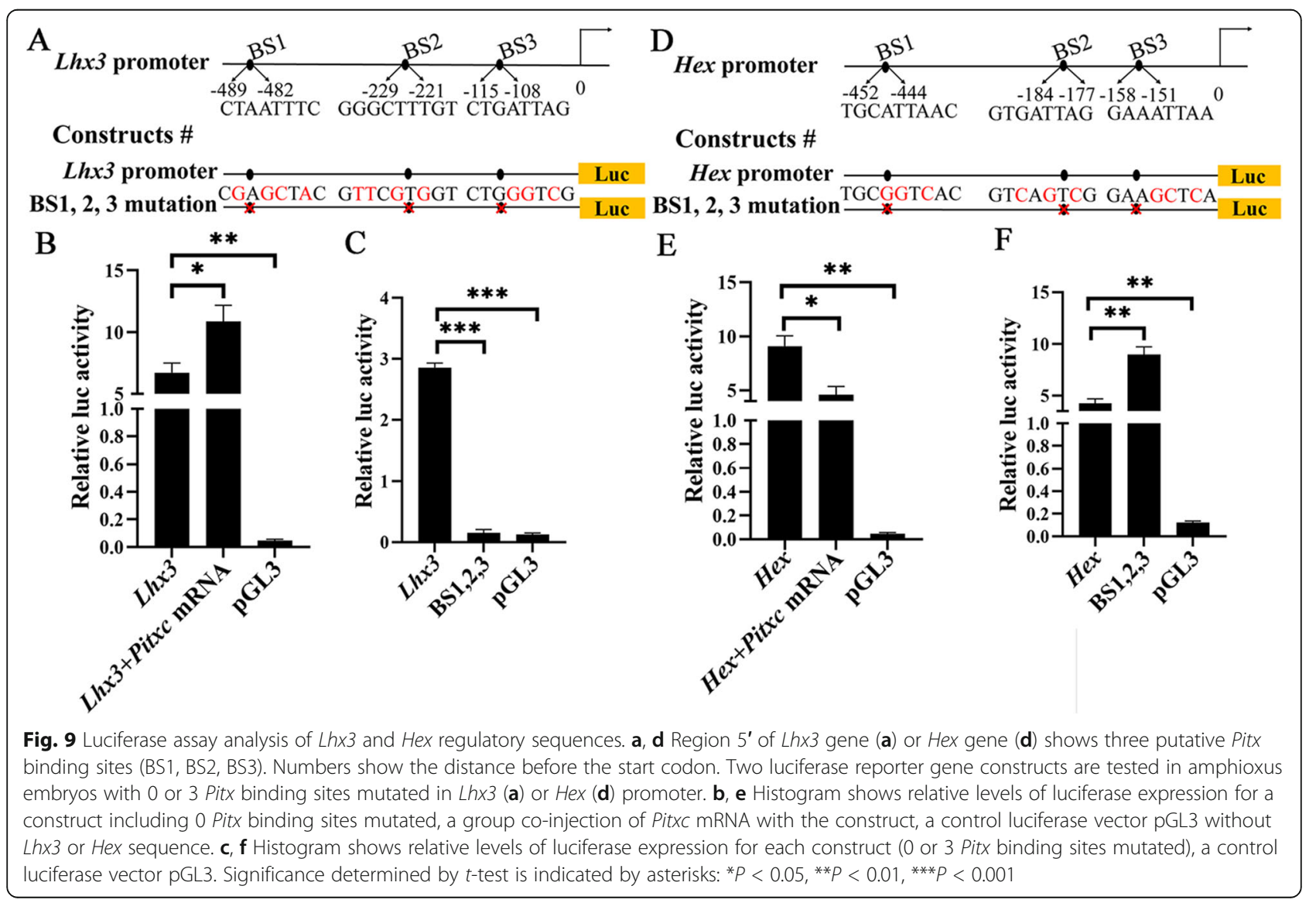


function has not been examined, although its asymmetric expression and regulation by Nodal signaling pathways have been reported in several invertebrate animals [24-28]. We here demonstrate that Pitx is required for LR morphogenesis in the chordate amphioxus. This indicates that involvement of Pitx in LR development predates chordate diversification, and also strengthens the importance of the gene in this process.

Although Pitx function in asymmetric development is generally conserved among chordates, the dependence of the gene in their LR morphogenesis is apparently different. In amphioxus, Pitx mutation affects asymmetries of most if not all LR organs, and causes loss of all left-side organs and ectopic formation of right-side structures on the left side. Whereas Pitx2 deficiency in zebrafish leads to no (or very weak) LR phenotype as mentioned above, and Pitx2 loss-of-function in mice causes right isomerism of the lungs and atrium, and defects in other visceral organs, but the position of the stomach and heart looping remain unaffected $[8,51-55]$. These differences reflect that LR organogenesis in amphioxus relies more heavily on Pitx than that in vertebrates, and that LR organogenesis in vertebrates may involve more regulatory factors.

Pitx $2 c$ is the only isoform of Pitx2 showing an asymmetric expression pattern in vertebrates [34]. Mice specially lacking asymmetric Pitx $2 c$ expression manifest LR defects comparable to Pitx knockout mice $[8,11]$, indicating that Pitx2c is a major if not the only player out of the three Pitx2 isoforms in mouse LR development. Similarly, we identified two Pitx isoforms in amphioxus: Pitxa/b and Pitxc, among which the amphioxus Pitxc showed asymmetric expression in embryos while Pitxa/b did not. However, unlike Pitx $2 c$ in mice, Pitxc mutation in amphioxus does not cause any LR defects. One possible interpretation of this discrepancy is that the mutation we introduced is hypomorphic. Indeed, Pitxa/c homozygotes from a cross between Pitxc heterozygotes and Pitx heterozygotes manifested LR defects at the larval stages, and importantly these defects were much milder compared to that observed in Pitx ${ }^{-/-}$mutants. Furthermore, there are two alternative in-frame start codons immediately after the mutation site we introduced in the Pitxc locus (Additional file 1: Table S2). These findings suggest that the Pitxc ${ }^{-/-}$mutants may use one of the two alternative start codons to make a dampened version of Pitxc protein. Interestingly, Pitxc expression was slightly upregulated in $\mathrm{Pitxc}^{-1-}$ mutants (Additional file 1: Fig. S14d) compared to their WT/Pitx ${ }^{-/-}$sibling (Additional file 1: Fig. S14c), while Pitxa/b expression remained unchanged (Additional file 1: Fig. S14a, b). We suggest this upregulation can, to some extent, compensate dampened Pitxc function and contribute toward normal development of Pitxc $^{-1-}$ embryos.
Our study provides several lines of evidence indicating that Pitx controls amphioxus LR morphogenesis by promoting development of organs on the left side and repressing formation of organs on the right side. First, Pitx mutation in amphioxus resulted in loss of left-side organs and ectopic formation of right-side organs on the left side. Second, genes essential for left-side organs like Lhx 3 and Prop 1 were activated after Pitx expression and their expression was observed in the region where Pitx was highly expressed, while genes required for the formation of organs on the right side, at least for the endostyle and CSG, were initiated either earlier (like Hex) or later (like FoxE4) than Pitx, and their expression was observed in the region where Pitx was not expressed. Importantly, the right-side genes were initially symmetrically expressed, and they became asymmetrically expressed (decreased expression on the left side) only when Pitx expression expanded into their expression domain. Third, while Pitx ${ }^{-/-}$mutants showed no expression of left-side genes and nearly bilaterally symmetric expression of right-side genes, Pitx overexpression induced expression of the left-side genes and inhibited that of the right-side genes. Fourth, Hex and $L h x 3$ genes, upstream regulators of the endostyle and preoral pit, respectively, had Pitx binding sites in their regulatory sequences, and mutation of these sites increased the activity of the Hex sequence, but decreased that of the Lhx3 sequence. Lastly, although BMP signaling pathway was thought to be a determinant of right-side development in vertebrates [20], our results demonstrated that this was not the case in amphioxus, as inhibition of BMP signaling after the N1 stage did not affect the asymmetrical expression pattern of Pitx, but reduced its expression on the left side (Additional file 1: Fig. S15d-i, $\left.\mathrm{d}^{\prime}-\mathrm{i}^{\prime}\right)$. However, it should be noted that blocking the BMP signal from late gastrula stage in amphioxus embryos eliminated Pitx expression on both sides (Additional file 1: Fig. S15b, b', c, c'), which is probably caused by ectopic induction of Nodal inhibitor Dand5 on the left side [56]. We are uncertain if vertebrates use a similar mechanism to pattern their LR organs in general, but we speculate that this may be the case in lungs and atria since Pitx2 is expressed in the left side of these organs in mice, and its deficiency results in right isomerism of these organs at the expense of their left-sided structures $[8,51-55]$. Consistent with this, a previous study showed that Pitx2c inhibited the development of sinoatrial node (a structure derived from the right atrium) by directly repressing Shox2, a transcriptional regulator of sinoatrial node gene program [57].

We showed that, as in vertebrates [3], LR morphogenesis in amphioxus required Pitx-independent signals (factor X) downstream of Nodal signaling. Bmp2/4 is recently shown to be asymmetrically expressed in 
amphioxus embryos from $\mathrm{N} 1$ to $\mathrm{N} 2$ stage in a $\mathrm{L}>\mathrm{R}$ manner, and inhibition of Nodal signaling at the gastrula stage eliminates $B m p 2 / 4$ expression [56]. This suggests a possibility for $B m p 2 / 4$ to be the $\mathrm{X}$ factor. To test this, we treated Pitx mutants with BMP inhibitor DMH1 from N0 to N5 stage or N0 to T1 stage and assayed the expression of Krox (marker of the glandular region of the CSG), $N k x 2.1$ (marker of the endostyle), and m-actin (marker of the somites). The treatment abolished the expression of the Krox gene in Pitx ${ }^{-/-}$mutants and their siblings at the N5 stage (Additional file 1: Fig. S16m, n), indicating that BMP signaling pathway was required for Krox expression. However, the treatment did not affect the expression of $N k \times 2.1$ and $m$-actin in either Pitx ${ }^{-/-}$ mutants (Additional file 1: Fig. S16p, t) or their siblings (Additional file 1: Fig. S16o, s). As a positive control, the treatment of mutants with the Nodal signaling inhibitor SB505124 at the same stages caused symmetric expression of Krox and $N k x 2.1$ in all examined embryos (Pit ${ }^{-/-}$mutants and their sibling) (Additional file 1: Fig. S16e-h). NOTCH, FGF, and RA play essential roles in the generation of somites from the tailbud of vertebrate embryos [33, 58]. Specifically, inhibition of RA signaling activity in zebrafish, mouse, and chicken led to asymmetric somites $[59,60]$. Different from vertebrates, RA and FGF signaling are not required for the formation and asymmetric patterning of somites from the tailbud in amphioxus [33]. However, this does not exclude the possibility of the signals as the $\mathrm{X}$ factor. To test this, we conducted similar experiments as described above and found that none of them appeared to be the $\mathrm{X}$ factor as treatment of Pitx mutants with inhibitors of NOTCH (DAPT), FGF (SU5402), and RA (BMS493) signaling pathways did not affect the expression patterns of $m$ actin (Additional file 1: Fig. S17k-p) and Nkx2.1 (Additional file 1: Fig. S16c-h) genes. Further studies are required to identify the nature of the $\mathrm{X}$ signal in the future.

The homologous relationship between the amphioxus preoral pit and vertebrate adenohypophysis was first indicated by anatomical studies [61], and later strengthened by gene expression data $[43,62]$. We here demonstrate that similar to vertebrates [63], Pitx is able to direct the development of amphioxus preoral pit through controlling expression of $L h \times 3$ and/or Prop1 genes. This further confirms the homologous relationship of the two organs. However, it is striking that the preoral pit is located on the left side of amphioxus while the adenohypophysis is formed at the anterior midline region of vertebrate embryos $[43,64]$. As Pitx is a master regulator of the two organs, we speculate that this difference is probably related to changes in Pitx expression between amphioxus and vertebrates. Previous studies showed that inhibition of Nodal signaling before $8 \mathrm{~S}$ stages abolished Pitx expression in amphioxus embryos [24]. We here further showed that Pitx expression relies on the Nodal signaling pathway at least until stage T1. This demonstrates that Nodal signaling is absolutely required for Pitx expression in amphioxus not only in the LR patterning phase, but also in early stage of LR morphogenesis. Vertebrates have evolved three Pitx genes (Pitx1, Pitx2 and Pitx3) by two rounds of whole genome duplications after their divergence from cephalochordates [65]. Among them, Pitx1 and Pitx2 are expressed in the adenohypophysis and are both required for its development $[66,67]$. However, their expressions in the adenohypophysis are regulated by signaling pathways other than Nodal [68]. Acquirement of Nodalindependent regulation of Pitx genes during vertebrate evolution is therefore likely a key event enabling the shift of the adenohypophysis to the midline in vertebrates.

Somites are unique to amphioxus and vertebrates within the chordates. Although vertebrate somites and the posterior somites of amphioxus are both generated from the tailbud, their formation mode and the patterning of them along the LR axis are different. In vertebrates, somites are formed symmetrically under a clock and wavefront mechanism [33, 58], whereas amphioxus lacks the mechanism and develops the posterior somites asymmetrically [69]. RA signal is required for symmetric somitogenesis of vertebrates through buffering the lateralizing influence of the LR machinery [59, 60]. Application of RA or BMS009 (an antagonist of the RA receptor) does not affect the asymmetric patterning of amphioxus somites, indicating RA signaling pathway is not involved in the process [33]. However, different from vertebrates, Pitx is continuously expressed in the left side of tailbud from the 8-somite stage during amphioxus development, and this expression relies strictly on the Nodal activity there. Moreover, inhibition of the Nodal activity after 8-somite stages or knockout of Pitx affects the asymmetry of amphioxus somites. Therefore, Nodal signal and Pitx, but not RA signal, are responsible for the asymmetric generation of somites in amphioxus. Considering that vertebrates have evolved a much more sophisticated process in somitogenesis than amphioxus, we speculate that the acquisition of RA signal, together with the loss of Nodal-Pitx cascade, in vertebrates might be the cause for their symmetric somitogenesis.

\section{Conclusions}

In summary, our study reveals that Pitx is a dominant (but not the only) factor downstream of Nodal signaling, in the LR morphogenesis of amphioxus (Fig. 10). During the development of amphioxus pharyngeal organs, Pitx promotes the development of left-side organs by directly activating expression of genes like $L h x 3$ (and/or Prop1 and Pit) (Fig. 10). Simultaneously, it acts together with 


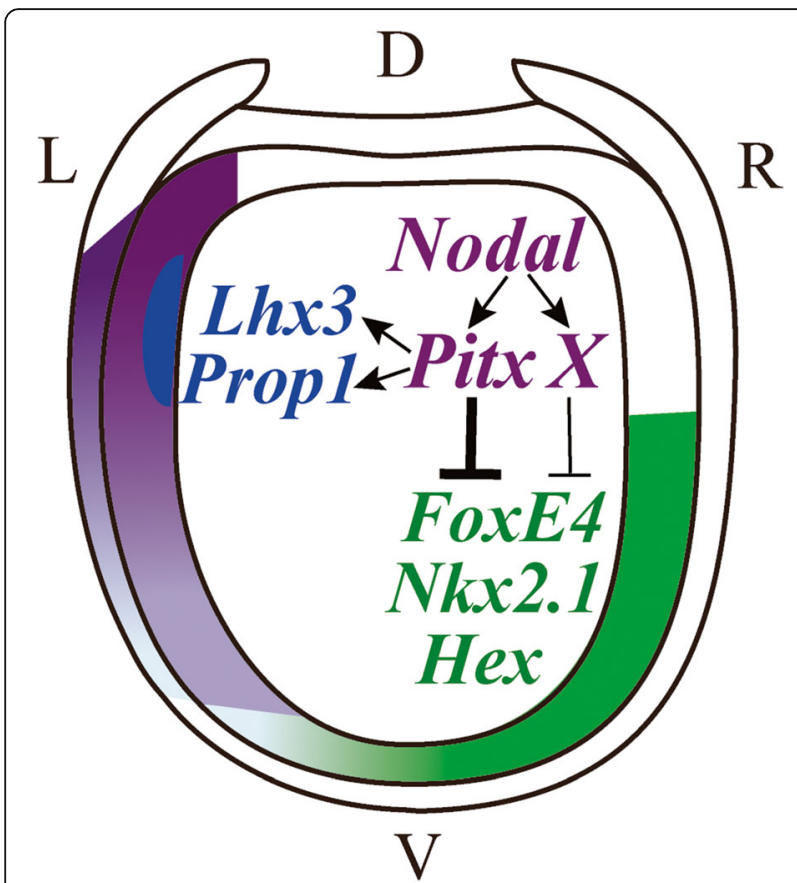

Fig. $10 \mathrm{~A}$ model for Pitx controlling the asymmetry of pharyngeal organs. At early neurula stage (before N4 stage), Pitx expression (gradient purple region) is in a gradient manner along the dorsalventral axis. During the process, Pitx promotes the development of organs on the left through directly activating expression of $L h \times 3$, Prop1, or/and Pit (blue region). Meanwhile, Pitx is a dominant factor together with an unknown factor $X$, and both factors are located downstream of Nodal signaling pathway, inhibiting the formation of right-side organs by directly repressing expression of Hex, Nkx2.1, or/ and FoxE4 (gradient green region) on the left side. D, dorsal; V, ventral; $L$, left side; $R$, right side

an unidentified factor X, also downstream of Nodal signaling, to inhibit formation of right-side organs by directly repressing expression of genes like Hex (and/or Nkx2.1 and FoxE4) in a dosage manner (Fig. 10). This provides essential clues for understanding the mechanisms of Pitx 2 in the regulation of asymmetric organogenesis in vertebrates, and also sheds important insights on why some organs like adenohypophysis are asymmetrically positioned in the amphioxus, but are symmetrically placed in vertebrates.

\section{Methods}

\section{Animals and embryo cultivation}

Amphioxus Branchiostoma floridae were obtained from Dr. Jr-Kai Yu's laboratory at the Institute of Cellular and Organismic Biology, Academia Sinica, Taiwan. They were maintained and induced to spawn following the protocol as we described and used in B. belcheri [70, 71], which is slightly different from the protocol recently developed for B. floridae [37]. Egg fertilization and embryo culture were carried out according to our previous report [72]. Embryos are staged according to a recent study unless otherwise stated [73, 74].

\section{Isolation of Pitx isoforms in amphioxus}

Pitxc isoform sequence was assembled from in-house generated transcriptome data [75], and Pitxa/b isoform (accession number: FE578121) was acquired by searching publicly available EST data in the NCBI database using Blast with the Pitxc sequence as the query. Three primers, two located in the first exons of Pitxc and Pitxa/b and one located in their common region (2nd exon of Pitxc and 3rd exon of Pitxa/b) were designed to verify their existence in amphioxus embryos. Exon/intron structures of the two isoforms were determined by aligning their mRNA sequences to the corresponding genome sequence on UCSC (genome-asia.ucsc.edu).

\section{Mutant generation and genotyping}

Generation and detection of Pitx mutants was conducted using the TALEN method as previously described [24, 36, 37, 75]. TALEN target sites of Pitx and mutation types used in the study are shown in Fig. 1. Primers for amplification of the flanking regions of the Pitx target sites and their amplicon sequences and sizes are listed in Additional file 2: Table S1. Genotyping of live embryos and embryos examined by whole mount in situ hybridization was performed as previously described [32, 37].

\section{Whole-mount in situ hybridization, histology, and immunofluorescence analysis}

pGEM-T-Easy constructs containing Cer, Nodal, Lefty, Pitxc, Lhx3, Krox, FoxE4, Nkx2.1, Pit, Pou4, m-actin, $M o p$, and $P a x 2 / 5 / 8$ mRNA sequences were obtained in previous studies [24, 31, 76, 77]. pGEM-T-Easy constructs containing partial or complete mRNA sequences of Hex, Prop1, Gata1/2/3, Pdvegfr, Scl, Hand, Pitxa/b, and Pitxc genes were constructed using protocols as previously reported [24, 31]. Primers used for gene cloning are listed in . Digoxigenin (DIG)-labeled antisense riboprobes (Roche) of all above genes were synthesized with Sp6 or T7 RNA polymerase (Thermo). Sequences are deposited in GenBank and their accession numbers are available in Additional file 2: Table S2.

Embryos at desired stages were fixed overnight with $4 \%(\mathrm{wt} / \mathrm{vol})$ PFA-MOPS-EGTA (pH 7.5) at $4{ }^{\circ} \mathrm{C}$, and then stored at $-20^{\circ} \mathrm{C}$ in $70 \%$ ethanol (vol/vol) for use. Whole-mount in situ hybridization (WISH) was performed following the description by $\mathrm{Yu}$ and Holland [78]. For the three Pitx gene probes, the one (called Pitx probe) transcribed from Pitxc isoform (indicated by the green line in Fig. 1a) was used for most experiments unless otherwise stated. Stained embryos were photographed using an inverted microscope (Olympus, IX71). After imaging, embryos were embedded in agarose- 
paraffin, sectioned at $5 \mu \mathrm{m}$ with a Leica RM2016 microtome, and then photographed using a brightfield microscope (Zeiss, AX10).

Acetylated $\alpha$-tubulin immunofluorescence staining for detection of peripheral nerves was performed as previously described [32]. Before staining, embryos and larvae were de-ciliated by a short incubation in $0.83 \mathrm{M} \mathrm{NaCl}$ seawater and then fixed in $4 \%$ (wt/vol) PFA-MOPSEGTA (pH 7.5) at $4{ }^{\circ} \mathrm{C}$ overnight [32]. DAPI (Invitrogen, $1 \mu \mathrm{g} / \mathrm{ml}$ in PBST) was used for nuclear staining. Images of stained embryos were acquired using a Zeiss LSM 780 confocal microscope.

\section{Quantification and statistical analysis}

The distance between somites or peripheral axons was measured using ImageJ software. Scatter boxplots were drawn using OriginPro (OriginLab Corporation). Statistical significance was tested by independent-samples $t$ tests with SPSS Statistics version 19 (IBM Corporation).

\section{mRNA synthesis and embryo microinjection}

A pXT7 construct containing Pitxc partial 5'UTR (207-bp), full-length coding sequence, and partial 3'UTR (217-bp) was conserved in a previous study [24]. Pitxc mRNAs was synthesized from the construct with T7 mMESSAGE mMACHINE kit (Ambion) following the kit manual. The mRNAs (300 ng/ $\mu \mathrm{L}$ ), together with $3 \mathrm{mg} / \mathrm{ml}$ fluorescein-labeled dextran (10,000 MW, Thermo Fisher Scientific) and 12.5\% glycerol, were injected into amphioxus unfertilized eggs or one blastomere of 2-cell embryos following steps described in previous reports [72].

\section{Luciferase activity assays}

Regulatory sequences of $L h x 3$ (a 500-bp fragment upstream of its transcriptional start) and Hex (a 554-bp fragment upstream of the transcriptional start) genes were determined according to the ATAC-seq data of $B$. lanceolatum [79]. They were respectively inserted upstream of the firefly luciferase gene in pGL3 (Promega) and modified by PCR to generate mutant versions (Additional file 2: Table S3). Pitx binding sites in the regulatory sequences of $L h x 3$ and Hex were predicted using JASPAR profiles at http://jaspar.genereg.net/. Injection solutions were prepared to contain $60 \mathrm{ng} / \mu \mathrm{L}$ of $L h x 3$ or Hex constructs, or Renilla luciferase vector pRL-SV40 (control), and $3 \mathrm{mg} / \mathrm{ml}$ fluorescein-labeled dextran (10,000 MW, Thermo Fisher Scientific), 12.5\% glycerol, with or without $100 \mathrm{ng} / \mu \mathrm{L}$ Pitxc mRNA. Embryos were injected at unfertilized egg stage [72] and assayed at N4 stage (eight somites, 60 embryos for each experiment); uninjected embryos from the same batch were used as the negative control. Firefly luciferase expression levels from pGL3 and Renilla luciferase from pRL-SV40 were detected with the Dual Luciferase Kit (Promega) using a
GloMax luminometer with an integration of $15 \mathrm{~s}$, and the level of firefly luciferase was normalized to Renilla luciferase activity for each experiment.

\section{Pharmacological treatments}

SB505124 (Sigma), dorsomorphin homolog-1 (DMH1, Sigma), DAPT (Calbiochem), SU5402 (Calbiochem), and BMS593 (Sigma) were used respectively to inhibit Nodal, BMP, NOTCH, FGF, and RA signaling pathway. They were dissolved in DMSO following standard protocols and used at the following concentrations: $50 \mu \mathrm{M}$ for SB505124, $40 \mu \mathrm{M}$ for DMH1, $150 \mu \mathrm{M}$ for DAPT, $50 \mu \mathrm{M}$ for SU5402, and $1 \mu \mathrm{M}$ for BMS593. SB505124 treatment was conducted at N0 (neurula with zero somite), $8 \mathrm{~S}$, 10S, T0, and T1 stages; DMH1, DAPT, SU5402, and BMS593 treatments were all conducted at N0 stage. For each treatment, an equal volume of DMSO was applied to the same batch of embryos and used as a negative control. All treatments were performed in six-well plates containing $3 \mathrm{~mL}$ of filtered seawater and 200-300 embryos. The treated embryos were fixed at required stages for in situ hybridization or cultured continuously for morphological observation.

\section{Supplementary Information}

The online version contains supplementary material available at https://doi. org/10.1186/s12915-021-01095-0.

Additional file 1: Figure S1. Location of Pitxa/c positive cells. Figure S2. Mutation rates of PitX TALEN1, TALEN2 and TALEN3 in F0 embryos. (Figure S3, S4 and S5) Genotype analysis of Pitx TALEN1, TALEN2 or TALEN3 mutants. Figure S6. The expression pattern of Dand5-NodalLefty-Pitx in Pitx mutants. Figure S7. The expression pattern of Pax2/5/8 in Pitx mutants. Figure S8. The dependence of Pitx gene on Nodal signaling. (Figure S9 and S10). Genotype analysis of Pitxa/C homozygotes carrying mutations at TALEN1 and TALEN2 or TALEN3. (Figure S11, S12 and S13)-Expression pattern of Pitx and asymmetrical pharyngeal organ markers in wildtype embryos and Pitx mutants. Table S1. DNA sequence upstream of start codon of $L h x 3$ and Hex gene. Table S2. Pitxc sequence. Figure S14-Expression of Pitxa/b and Pitxc in Pitxc mutants. Figure S15. The dependence of Pitx gene on BMP signaling after N1 stage. (Figure S16 and S17) Studies for identifying the X factor.

Additional file 2: Table S1. Primers used for amplifying gene fragments including TALEN target sites and the amplicon sequences. Table S2. Primers used for making pGEM-T-Easy constructs. Table S3. Primers used for cloning and mutagenesis of $L h \times 3$ and Hex promoter sequence.

Additional file 3. Uncrooped gel images. Uncropped gel images shown in Additional file 1 figures.

\section{Acknowledgements}

We thank Dr. Qingming Qu and Yichen Dai for their critical reading of the manuscript, and Prof. Sebastian M. Shimeld for language editing.

\section{Authors' contributions}

CX, RP, GH, and XL generated amphioxus mutants; CX and RP performed most experiments; CX, RP, YW, and GL analyzed the data; GL designed experiments; GL and CX wrote the first draft of the manuscript. The authors read and approved the final manuscript. 


\section{Funding}

This work was supported by the National Natural Science Foundation of China (No. 31872186, No. 31672246, No. 32070458, and No. 31900370) and the Natural Science Foundation of Fujian Province of China (No. 2019 J01022).

\section{Availability of data and materials}

All relevant data are within the paper and its Supporting Information.

\section{Declarations}

Ethics approval and consent to participate

Not applicable

\section{Consent for publication}

Not applicable

\section{Competing interests}

The authors declare that they have no competing interests.

\section{Author details}

${ }^{1}$ State Key Laboratory of Cellular Stress Biology, School of Life Sciences, Xiamen University, Xiangan District, Xiamen 361102, Fujian, China. ${ }^{2} J i a n g s u$ Key Laboratory of Marine Biotechnology, School of Marine Science and Fisheries, Jiangsu Ocean University, Lianyungang 222005, China.

Received: 12 April 2021 Accepted: 13 July 2021

Published online: 20 August 2021

\section{References}

1. Hamada H, Meno C, Watanabe D, Saijoh Y. Establishment of vertebrate leftright asymmetry. Nat Rev Genet. 2002;3(2):103-13. https://doi.org/10.1038/ nrg732.

2. Blum M, Feistel $K$, Thumberger $T$, Schweickert $A$. The evolution and conservation of left-right patterning mechanisms. Development. 2014 141(8):1603-13. https://doi.org/10.1242/dev.100560.

3. Grimes DT, Burdine RD. Left-right patterning: breaking symmetry to asymmetric morphogenesis. Trends Genet. 2017;33(9):616-28. https://doi. org/10.1016/j.tig.2017.06.004.

4. Hamada H, Tam P. Diversity of left-right symmetry breaking strategy in animals. F1000Res. 2020;9:1123. https://doi.org/10.12688/f1000research.21 670.1 .

5. Nakamura $T$, Hamada $H$. Left-right patterning: conserved and divergent mechanisms. Development. 2012;139(18):3257-62. https://doi.org/10.1242/ dev.061606

6. Shiratori H, Sakuma R, Watanabe M, Hashiguchi H, Mochida K, Sakai Y, et al. Two-step regulation of left-right asymmetric expression of Pitx2: initiation by nodal signaling and maintenance by Nkx2. Mol Cell. 2001;7(1):137-49. https://doi.org/10.1016/s1097-2765(01)00162-9.

7. Lu M-F, Pressman C, Dyer R, Johnson RL, Martin JF. Function of Rieger syndrome gene in left-right asymmetry and craniofacial development. Nature. 1999:401(6750):276-8. https://doi.org/10.1038/45797.

8. Lin CR, Kioussi C, O'Connell S, Briata P, Szeto D, Liu F, et al. Pitx2 regulates lung asymmetry, cardiac positioning and pituitary and tooth morphogenesis. Nature 1999:401(6750):279-82. https://doi.org/10.1038/45803.

9. Yu X, St Amand TR, Wang S, Li G, Zhang Y, Hu Y, et al. Differential expression and functional analysis of Pitx2 isoforms in regulation of heart looping in the chick. Development. 2001;128(6):1005-13. https://doi.org/1 0.1242/dev.128.6.1005

10. Dagle JM, Sabel JL, Littig JL, Sutherland LB, Kolker SJ, Weeks DL. Pitx2C attenuation results in cardiac defects and abnormalities of intestinal orientation in developing Xenopus laevis. Dev Biol. 2003;262(2):268-81. https://doi.org/10.1016/s0012-1606(03)00389-0.

11. Liu CY, Liu W, Lu MF, Brown NA, Martin JF. Regulation of left-right asymmetry by thresholds of Pitx2C activity. Development. 2001;128(11): 2039-18.

12. Gage PJ, Suh H, Camper SA. Dosage requirement of Pitx2 for development of multiple organs. Development. 1999;126(20):4643-51. https://doi.org/1 0.1242/dev.126.20.4643

13. Liu W, Selever J, Lu M-F, Martin JF. Genetic dissection of Pitx2 in craniofacial development uncovers new functions in branchial arch morphogenesis, late aspects of tooth morphogenesis and cell migration. Development. 2003; 130(25):6375-85. https://doi.org/10.1242/dev.00849.

14. Welsh IC, Thomsen M, Gludish DW, Alfonso-Parra C, Bai Y, Martin JF, et al. Integration of left-right Pitx2 transcription and Wnt signaling drives asymmetric gut morphogenesis via Daam2. Dev Cell. 2013;26(6):629-44. https://doi.org/10.1016/j.devcel.2013.07.019.

15. Ji Y, Buel SM, Amack JD. Mutations in zebrafish pitx2 model congenital malformations in Axenfeld-Rieger syndrome but do not disrupt left-right placement of visceral organs. Dev Biol. 2016;416(1):69-81. https://doi.org/1 0.1016/j.ydbio.2016.1006.1010.

16. Liu Y, Semina EV. pitx2 deficiency results in abnormal ocular and craniofacial development in zebrafish. PLoS One. 2012;7(1):e30896. https://doi.org/1 0.31371/journal.pone.0030896.

17. Garric L, Ronsin B, Roussigné M, Booton S, Blader P. Pitx2C ensures habenular asymmetry by restricting parapineal cell number. Development. 2014;141(7):1572-9. https://doi.org/10.1242/dev.100305.

18. Brennan J, Norris DP, Robertson EJ. Nodal activity in the node governs leftright asymmetry. Genes Dev. 2002;16(18):2339-44. https://doi.org/10.1101/ gad.1016202.

19. Rago L, Castroviejo N, Fazilaty H, Garcia-Asencio F, Ocaña OH, Galcerán J, et al. MicroRNAs establish the right-handed dominance of the heart laterality pathway in vertebrates. Dev Cell. 2019;51(4):446-59. https://doi. org/10.1016/j.devcel.2019.09.012.

20. Ocaña OH, Coskun H, Minguillón C, Murawala P, Tanaka EM, Galcerán J, et al. A right-handed signalling pathway drives heart looping in vertebrates. Nature. 2017;549(7670):86-90. https://doi.org/10.1038/nature23454.

21. Castroviejo N, Ocaña OH, Rago L, Coskun H, Arcas A, Galcerán J, et al. Reply to: Zebrafish prrx1a mutants have normal hearts. Nature. 2020;585(7826): E17-9. https://doi.org/10.1038/s41586-020-2675-0.

22. Tessadori F, Bakker DEMD, Barske L, Nelson N, Bakkers J. Zebrafish prrx1a mutants have normal hearts. Nature. 2020;585(7826):E14-6. https://doi.org/1 0.1038/s41586-020-2674-1.

23. Namigai EKO, Kenny NJ, Shimeld SM. Right across the tree of life: the evolution of left-right asymmetry in the Bilateria. Genesis. 2014;52(6):45870. https://doi.org/10.1002/dvg.22748.

24. Li G, Liu X, Xing C, Zhang H, Shimeld SM, Wang Y. Cerberus-Nodal-Lefty-Pitx signaling cascade controls left-right asymmetry in amphioxus. Proc Natl Acad Sci U S A. 2017;114(14):3684-9. https://doi.org/10.1073/pnas.1620519114.

25. Morokuma J, Ueno M, Kawanishi H, Saiga H, Nishida H. HrNodal, the ascidian nodal-related gene, is expressed in the left side of the epidermis, and lies upstream of HrPitx. Dev Genes Evol. 2002;212(9):439-46. https://doi. org/10.1007/s00427-00002-00242-00423.

26. Duboc V, Röttinger E, Lapraz F, Besnardeau L, Lepage T. Left-right asymmetry in the sea urchin embryo is regulated by nodal signaling on the right side. Dev Cell. 2005;9(1):147-58. https://doi.org/10.1016/j.devcel.2005.1 005.1008

27. Grande C, Patel NH. Nodal signalling is involved in left-right asymmetry in snails. Nature. 2009;457(7232):1007-11. https://doi.org/10.1038/nature07603.

28. Soukup V, Yong LW, Lu T-M, Huang S-W, Kozmik Z, Yu J-K. The Nodal signaling pathway controls left-right asymmetric development in amphioxus. Evodevo. 2015;6:5. https://doi.org/10.1186/2041-9139-6-5.

29. Boorman CJ, Shimeld SM. The evolution of left-right asymmetry in chordates. Bioessays. 2002;24(11):1004-11. https://doi.org/10.1002/bies.10171.

30. Zhang H, Chen S, Shang C, Wu X, Li G. Interplay between Lefty and Nodal signaling is essential for the organizer and axial formation in amphioxus embryos. Dev Biol. 2019;456(1):63-73. https://doi.org/10.1016/j.ydbio.2019.1 008.1006

31. Hu G, Li G, Wang H, Wang Y. Hedgehog participates in the establishment of left-right asymmetry during amphioxus development by controlling Cerberus expression. Development. 2017;144(24):4694-703. https://doi.org/1 $0.1242 /$ dev. 157172 .

32. Zhu X, Shi C, Zhong Y, Liu X, Yan Q, Wu X, et al. Cilia-driven asymmetric Hedgehog signalling determines the amphioxus left-right axis by controlling Dand5 expression. Development. 2020;147(1):dev182469. https:// doi.org/10.1242/dev.182469.

33. Bertrand S, Aldea D, Oulion S, Subirana L, de Lera A, Somorjai I, et al. Evolution of the role of RA and FGF signals in the control of somitogenesis in chordates. PLoS One. 2015;10(9):e0136587. https://doi.org/10.1371/journal. pone.0136587.

34. Schweickert A, Campione M, Steinbeisser H, Blum M. Pitx2 isoforms: involvement of Pitx2c but not Pitx2a or Pitx2b in vertebrate left-right 
asymmetry. Mech Dev. 2000;90(1):41-51. https://doi.org/10.1016/s0925-4773 (99)00227-0.

35. Christiaen L, Bourrat F, Joly J-S. A modular cis-regulatory system controls isoform-specific pitx expression in ascidian stomodæum. Dev Biol. 2005; 277(2):557-66. https://doi.org/10.1016/j.ydbio.2004.10.008.

36. Li G, Feng J, Lei $Y$, Wang J, Wang $H$, Shang L-K, et al. Mutagenesis at specific genomic loci of amphioxus Branchiostoma belcheri using TALEN method. J Genet Genomics. 2014;41(4):215-9. https://doi.org/10.1016/j.jgg.2 014.1002.1003.

37. Holland LZ, Li G. In: Carroll DJ, editor. Developmental biology of the sea urchin and other marine invertebrates. New York: Humana; 2021. p. 1-29.

38. Pascual-Anaya J, Albuixech-Crespo B, Somorjai IML, Carmona R, GarciaFernàndez $\mathrm{J}$. The evolutionary origins of chordate hematopoiesis and vertebrate endothelia. Dev Biol. 2013;375(2):182-92. https://doi.org/10.1016/ j.ydbio.2012.1011.1015

39. Kaji T, Reimer JD, Morov AR, Kuratani S, Yasui K. Amphioxus mouth after dorso-ventral inversion. Zoological Lett. 2016;2(1):2. https://doi.org/10.1186/ s40851-016-0038-3

40. Kozmik Z, Holland ND, Kalousova A, Paces J, Holland LZ. Characterization of an amphioxus paired box gene, AmphiPax2/5/8: developmental expression patterns in optic support cells, nephridium, thyroid-like structures and pharyngeal gill slits, but not in the midbrain-hindbrain boundary region. Development. 1999;126(6):1295-304. https://doi.org/10.1242/dev.126.6.1295.

41. Lu TM, Luo YJ, Yu JK. BMP and Delta/Notch signaling control the development of amphioxus epidermal sensory neurons: insights into the evolution of the peripheral sensory system. Development. 2012;139(11): 2020-30. https://doi.org/10.1242/dev.073833.

42. Schubert M, Holland LZ, Stokes MD, Holland ND. Three amphioxus Wnt genes (AmphiWnt3, AmphiWnt5, and AmphiWnt6) associated with the tail bud: the evolution of somitogenesis in chordates. Dev Biol. 2001;240(1):26273. https://doi.org/10.1006/dbio.2001.0460.

43. Candiani S, Holland ND, Oliveri D, Parodi M, Pestarino M. Expression of the amphioxus Pit-1 gene (AmphiPOU1F1/Pit-1) exclusively in the developing preoral organ, a putative homolog of the vertebrate adenohypophysis. Brain Res Bull. 2008; 75(2-4):324-30. https:/doi.org/10.1016/j.brainresbull.2007.10.023.

44. Venkatesh TV, Holland ND, Holland LZ, Su M-T, Bodmer R. Sequence and developmental expression of amphioxus AmphiNk2-1: insights into the evolutionary origin of the vertebrate thyroid gland and forebrain. Dev Genes Evol. 1999;209(4):254-9. https://doi.org/10.1007/s004270050250.

45. Wang Y, Zhang PJ, Yasui K, Saiga H. Expression of Bblhx3, a LIM-homeobox gene, in the development of amphioxus Branchiostoma belcheri tsingtauense. Mech Dev. 2002;117(1-2):315-9. https://doi.org/10.1016/s092 5-4773(02)00197-1.

46. Angotzi AR, Mungpakdee S, Stefansson S, Male R, Chourrout D. Involvement of Prop 1 homeobox gene in the early development of fish pituitary gland. Gen Comp Endocrinol. 2011;171(3):332-40. https://doi.org/10.1016/j.ygcen.2 011.02 .026$.

47. Onai T, Lin H-C, Schubert M, Koop D, Osborne PW, Alvarez S, et al. Retinoic acid and Wnt/ß-catenin have complementary roles in anterior/posterior patterning embryos of the basal chordate amphioxus. Dev Biol. 2009;332(2): 223-33. https://doi.org/10.1016/j.ydbio.2009.1005.1571.

48. Yu JK, Holland LZ, Jamrich M, Blitz IL, Holland ND. AmphiFoxE4, an amphioxus winged helix/forkhead gene encoding a protein closely related to vertebrate thyroid transcription factor-2: expression during pharyngeal development. Evol Dev. 2002;4(1):9-15. https://doi.org/10.1046/j.1525-1142 x.2002.01057.x

49. Nilsson M, Fagman $H$. Development of the thyroid gland. Development 2017;144(12):2123-40. https://doi.org/10.1242/dev.145615.

50. Scully KM, Rosenfeld MG. Pituitary development: regulatory codes in mammalian organogenesis. Science. 2002;295(5563):2231-5. https://doi. org/10.1126/science.1062736.

51. Shiratori $H$, Shen $M$, Hamada $H$. Conserved regulation and role of Pitx2 in situs-specific morphogenesis of visceral organs. Development. 2006;295(1): 369-70. https://doi.org/10.1242/dev.02470.

52. Ryan AK, Blumberg B, Rodriguez-Esteban C, Yonei-Tamura S, Tamura K, Tsukui T, et al. Pitx2 determines left-right asymmetry of internal organs in vertebrates. Nature. 1998;394(6693):545-51. https://doi.org/10.1038/29004.

53. Yoshioka H, Meno C, Koshiba K, Sugihara M, Itoh H, Ishimaru Y, et al. Pitx2, a bicoid-type homeobox gene, is involved in a lefty-signaling pathway in determination of left-right asymmetry. Cell. 1998;94(3):299-305. https://doi. org/10.1016/s0092-8674(00)81473-7.
54. Piedra ME, Icardo JM, Albajar M, Rodriguez-Rey JC, Ros MA. Pitx2 participates in the late phase of the pathway controlling left-right asymmetry. Cell. 1998; 94(3):319-24. https://doi.org/10.1016/s0092-8674(00)81475-0.

55. Campione M, Steinbeisser H, Schweickert A, Deissler K, Blum M. The homeobox gene Pitx2: mediator of asymmetric left-right signaling in vertebrate heart and gut looping. Development. 1999;126(6):1225-34. https://doi.org/10.1242/dev.126.6.1225.

56. Soukup V, Kozmik Z. The Bmp signaling pathway regulates development of left-right asymmetry in amphioxus. Dev Biol. 2018;434(1):164-74. https://doi. org/10.1016/j.ydbio.2017.1012.1004.

57. Wang J, Klysik E, Sood S, Johnson RL, Wehrens XH, Martin JF. Pitx2 prevents susceptibility to atrial arrhythmias by inhibiting left-sided pacemaker specification. Proc Natl Acad Sci U S A. 2010;107(21):9753-8. https://doi. org/10.1073/pnas.0912585107.

58. Maroto M, Bone RA, Dale JK. Somitogenesis. Development. 2012;139(14): 2453-6. https://doi.org/10.1242/dev.069310.

59. Vermot J, Pourquie $O$. Retinoic acid coordinates somitogenesis and leftright patterning in vertebrate embryos. Nature. 2005;435(7039):215-20. https://doi.org/10.1038/nature03488.

60. Kawakami Y, Raya A, Raya RM, Rodríguez-Esteban C, Belmonte JCl. Retinoic acid signalling links left-right asymmetric patterning and bilaterally symmetric somitogenesis in the zebrafish embryo. Nature. 2005;435(7039): 165-71. https://doi.org/10.1038/nature03512.

61. Welsch LT, Welsch U. Histological and Elektronmicroscopical observations on the preoral ciliary groove of Saccoglossus horsti (Hemichordata) and on Hatschek's pit of Branchiostoma lanceolatum (Acrania). A contribution to the phylogenetic development of the adenohypophysis. Zool Jb Anat. 1978;100:564-78.

62. Glardon S, Holland LZ, Gehring WJ, Holland ND. Isolation and developmental expression of the amphioxus Pax-6 gene (AmphiPax-6): insights into eye and photoreceptor evolution. Development. 1998;125(14): 2701-10. https://doi.org/10.1242/dev.125.14.2701.

63. Tremblay J, Lanctôt C, Drouin J. The pan-pituitary activator of transcription, Ptx1 (pituitary homeobox 1), acts in synergy with SF-1 and Pit1 and is an upstream regulator of the Lim-homeodomain gene Lim3/Lhx3. Mol Endocrinol. 1998;12(3):428-41. https://doi.org/10.1210/mend.1212.1213.0073.

64. Kawamura K, Kouki T, Kawahara G, Kikuyama S. Hypophyseal development in vertebrates from amphibians to mammals. Gen Comp Endocrinol. 2002; 126(2):130-5. https://doi.org/10.1006/gcen.2002.7784.

65. Yasui K, Zhang SC, Uemura M, Saiga H. Left-right asymmetric expression of BbPtx, a Ptx-related gene, in a lancelet species and the developmental leftsidedness in deuterostomes. Development. 2000;127(1):187-95. https://doi. org/10.1242/dev.127.1.187

66. Gage PJ, Suh H, Camper SA. The bicoid-related Pitx gene family in development. Mamm Genome. 1999;10(2):197-200. https://doi.org/10.1007/ s003359900970.

67. Charles MA, Hoonkyo S, Hjalt TA, Jacques D, Camper SA, Gage PJ. PITX genes are required for cell survival and Lhx3 activation. Mol Endocrinol. 2005;19(7):1893-903. https://doi.org/10.1210/me.2005-0052.

68. Zhu X, Gleiberman AS, Rosenfeld MG. Molecular physiology of pituitary development: signaling and transcriptional networks. Physiol Rev. 2007; 87(3):933-63. https://doi.org/10.1152/physrev.00006.

69. Beaster-Jones L, Kaltenbach SL, Koop D, Yuan S, Chastain R, Holland LZ. Expression of somite segmentation genes in amphioxus: a clock without a wavefront? Dev Genes Evol. 2008;218(11):599-611. https://doi.org/10.1007/ s00427-008-0257-5.

70. Li G, Shu Z, Wang Y. Year-round reproduction and induced spawning of Chinese amphioxus, Branchiostoma belcheri, in laboratory. PLoS One. 2013; 8(9):e75461. https://doi.org/10.71371/journal.pone.0075461.

71. Li G, Yang X, Shu Z, Chen X, Wang Y. Consecutive spawnings of Chinese amphioxus, Branchiostoma belcheri, in captivity. PLoS One. 2012;7(12): e50838. https://doi.org/10.51371/journal.pone.0050838.

72. Liu X, Li G, Feng J, Yang X, Wang Y-Q. An efficient microinjection method for unfertilized eggs of Asian amphioxus Branchiostoma belcheri. Dev Genes Evol. 2013;223(4):269-78. https://doi.org/10.1007/s00427-013-0441-0.

73. Carvalho JE, Lahaye F, Yong LW, Croce JC, Escrivá H, Yu J-K, et al. An updated staging system for cephalochordate development: one table suits them all. Front Cell Dev Biol. 2021;9:668006. https://doi.org/10.3389/fcell.2 021.668006

74. Bertrand S, Carvalho J, Dauga D, Matentzoglu N, Daric V, Yu J-K, et al. The ontology of the amphioxus anatomy and life cycle (AMPHX). Front Cell Dev Biol. 2021;9:668025. https://doi.org/10.3389/fcell.2021.668025 
75. Qin Y, Lin Z, Shi D, Zhong M, An T, Chen L, et al. Integrate heterogeneous NGS and TGS data to boost genome-free transcriptome research. bioRxiv. 2020; http://www.bio-add.org/InTrans/.

76. Ren Q, Zhong Y, Huang X, Leung B, Li G. Step-wise evolution of neural patterning by Hedgehog signalling in chordates. Nat Ecol Evol. 2020;4(9): 1247-55. https://doi.org/10.1038/s41559-020-1248-9.

77. Zhong Y, Herrera-Beda C, Garcia-Fernàndez J, Li G, Holland PWH. Mutation of amphioxus $P d x$ and $C d x$ demonstrates conserved roles for ParaHox genes in gut, anus and tail patterning. BMC Biol. 2020;18(1):68. https://doi.org/1 0.1186/s12915-020-00796-2.

78. Yu J, Holland L. Amphioxus whole-mount in situ hybridization. Cold Spring Harb Protoc. 2009;2009(9):pdb. prot5286. https://doi.org/10.1101/pdb.prot52 86http://doi.org/20147271.

79. Marlétaz F, Firbas P, Maeso I, Tena JJ, Bogdanovic O, Perry M, et al. Amphioxus functional genomics and the origins of vertebrate gene regulation. Nature; UCSC https://genome-asia.ucsc.edu/cgi-bin/hgBla t?hgsid=733042239_ZKPiACVsSpsLOmlaCe5abNzQg7Dn\&command=star; 2018

\section{Publisher's Note}

Springer Nature remains neutral with regard to jurisdictional claims in published maps and institutional affiliations.

Ready to submit your research? Choose BMC and benefit from:

- fast, convenient online submission

- thorough peer review by experienced researchers in your field

- rapid publication on acceptance

- support for research data, including large and complex data types

- gold Open Access which fosters wider collaboration and increased citations

- maximum visibility for your research: over $100 \mathrm{M}$ website views per year

At BMC, research is always in progress.

Learn more biomedcentral.com/submissions 